\title{
A New Class of Two-Channel Biorthogonal Filter Banks and Wavelet Bases
}

\author{
See-May Phoong, Student Member, IEEE, Chai W. Kim, P. P. Vaidyanathan, Fellow, IEEE, and Rashid Ansari
}

\begin{abstract}
We propose a novel framework for a new class of two-channel biorthogonal filter banks. The framework covers two useful subclasses:

i) causal stable IIR filter banks

ii) linear phase FIR filter banks.

There exists a very efficient structurally perfect reconstruction implementation for such a class. Filter banks of high frequency selectivity can be achieved by using the proposed framework with low complexity. The properties of such a class are discussed in detail. The design of the analysis/synthesis systems reduces to the design of a single transfer function. Very simple design methods are given both for FIR and IIR cases. Zeros of arbitrary multiplicity at aliasing frequency can be easily imposed, for the purpose of generating wavelets with regularity property. In the IIR case, two new classes of IIR maximally flat filters different from Butterworth filters are introduced. The filter coefficients are given in closed form. The wavelet bases corresponding to the biorthogonal systems are generated. We also provide a novel mapping of the proposed 1-D framework into 2-D. The mapping preserves the following:

i) perfect reconstruction

ii) stability in the IIR case

iii) linear phase in the FIR case

iv) zeros at aliasing frequency

v) frequency characteristic of the filters.
\end{abstract}

\section{INTRODUCTION}

F IG. 1(a) shows a two-channel maximally decimated filter bank, and Fig. 1(b) shows the well-known polyphase form for this system. The applications of such multirate systems are well-known [1]-[7]. If for all input $x(n)$, the output of the system $\widehat{x}(n)=c x\left(n-n_{0}\right)$ for some nonzero constant $c$ and integer $n_{0}$, the system is called a perfect reconstruction (PR) system. In the maximally decimated case, $P R$ is equivalent to biorthogonality [5]. A number of PR or nearly PR systems have been reported before. In this paper we develop several new results for two-channel biorthogonal filter banks based on a useful class of polyphase matrices.

\section{A. Previous Work}

In FIR filter banks, all the four filters $H_{0}, H_{1}, F_{0}$, and $F_{1}$, are FIR filters while in the case of IIR filter banks, some or all

Manuscript received October 8, 1993; revised August 18, 1994. This work was supported by Office of Naval Research under Grant N00 014-93-0231 and funds from Tektronix, Inc. The associate editor coordinating the review of this paper and approving it for publication was Prof. Roberto H. Bamberger.

S.-M. Phoong and P. P. Vaidyanathan are with the Department of Electrical Engineering, California Institute of Technology, Pasadena, CA, 91125 USA.

C. W. Kim is with the Department of Electrical Engineering, University of Pennsylivania, Philadelphia, PA, 19104 USA.

R. Ansari is with Bellcore MRE, Morristown, NJ, 07960 USA.

IEEE Log Number 9408226.

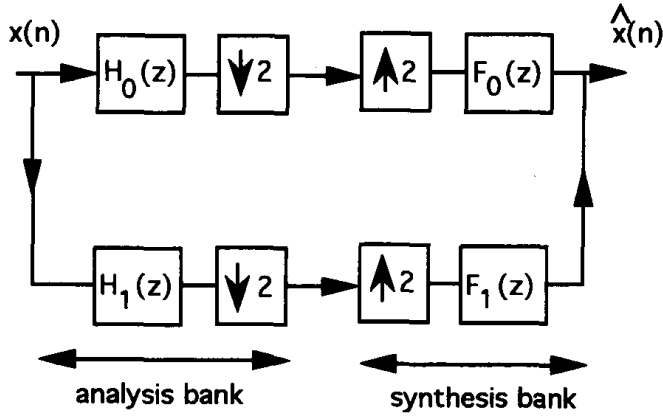

(a)

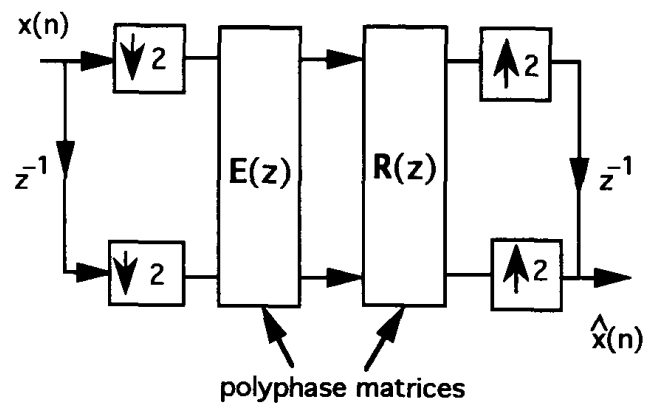

(b)

Fig. 1. (a) Two-channel analysis/synthesis filter bank; (b) redrawing of (a) by using the polyphase representation.

of these filters are IIR filters. The earliest good designs for the IIR case were such that the analysis bank was paraunitary and the polyphase components of $H_{0}(z)$ and $H_{1}(z)$ were allpass (see [7], and p. 201 of [2]). Even though all the IIR filters are causal stable, the reconstructed signal suffers from phase distortion. IIR PR filter banks typically have noncausal stable filters or causal unstable filters [8]-[10]. Recently the authors in [11] proposed a IIR PR technique providing causal stable solutions, but no satisfactory design method was given.

In earlier design of 2-D filter banks, separable filters have been considered because of their advantage of low complexity. However nonseparable filters offer more freedom in the design and hence in general will give better performance. Recently, some results on the nonseparable filter banks have emerged [12]-[14]. However, few design techniques are available for nonseparable PR filter banks. In [12], a design method based on space domain approach is given. In [13], a subclass of 2-D paraunitary systems (which can be represented as a cascade of 1-D paraunitary systems of degree one) is 
considered. However, in both of the polyphase approaches above, the optimization in the designs involves a large number of nonlinear constraints. Thus other approaches, such as 1D to 2-D mapping, have been considered [14]-[19]. In [14], even though PR property is preserved by the mapping, the frequency responses of the filters will change. In [15] and [16], a mapping of 1-D filter banks to 2-D filter banks is given. The authors apply the technique on a 1-D twochannel orthogonal IIR system to achieve a 2-D IIR filter bank. The resulting systems have either phase distortion or stability. problem. In [17], the authors employ McClellan's transformation on the 1-D maximally flat FIR halfband filters to obtain a 2-D biorthogonal filter bank. However, because of the lack of factorization theorems in the 2-D case, one of the lowpass filters is constrained to have all its zeros at the aliasing frequency. In addition, there is no simple way to ensure the frequency selectivity of all the filters. In [18], the authors introduce a mapping which can be viewed as the generalization McClellan's transformation. 2-D two-channel PR systems with good frequency selectivity can be obtained by judiciously designing the mapping. However, the mapping works for the FIR case only and the resulting filters usually have a large number of coefficients.

Some of the results in this paper were reported in the earlier conference papers [20]-[22]. For the 1-D case [20], both of the linear phase FIR and causal stable IIR solutions for PR filter banks similar to those proposed in this paper were given. For the 2-D quincunx case [21], the authors constructed a 1-D to 2-D mapping (which is the same as the mapping given in Section $V$ in this paper) that preserved many of the desired properties. However many of the properties of the 1-D and 2D biorthogonal systems were not addressed in [20], [21], for example, the problem of imposition of zeros at the aliasing frequency which is important for the purpose of generating smooth wavelet basis functions.

\section{$B$. The New Idea and Its Merits}

In this paper, we constrain the polyphase matrix $\mathbf{E}(z)$ such that $[\operatorname{det} \mathbf{E}(z)]$ is a delay. Furthermore, we consider $\mathbf{E}(z)$ and $\mathbf{R}(z)$ to be either i) both causal stable IIR or ii) both FIR. In each case, the following properties can be simultaneously satisfied:

1) Perfect reconstruction is preserved structurally and the structural complexity is very low.

2) All analysis and synthesis filters are designed by controlling a single transfer function $\beta(z)$ [allpass in the IIR case, and Type 2 (i.e., odd order symmetric linear phase FIR) in the FIR case]. So the design procedure is very simple. It is very easy to design $\beta(z)$ so that all filters have good responses (lowpass or highpass as the case may be).

3) In the IIR case, all the analysis and synthesis filters are causal and stable.

4) In some applications such as image coding, the linear phase property of the analysis and synthesis filters is desired. In the FIR case, the filters are exact linear-phase. In the IIR case, we can force the phase response of the filters to be nearly linear in the passband, as we shall explain and demonstrate.

5) The lowpass analysis filter $H_{0}(z)$ can be forced to have arbitrary number of zeros at $\omega=\pi$. Furthermore the lowpass synthesis filter $F_{0}(z)$ is guaranteed to have the same number of zeros at $\pi$ as $H_{0}(z)$. In both of the IIR and FIR cases, we give closed form expression for the filter coefficients that provide maximum number of zeros at $\pi$.

A new class of biorthogonal wavelet basis functions can be generated from the above filter bank. The regularity property can be directly controlled by imposing multiple zeros at $\pi$ as desired. In the IIR case, since all filters are causal (in addition to being stable), the basis functions are all causal. In the FIR case, the linear phase property ensures symmetry of the wavelets, while at the same time providing a simple control on regularity (because the number of zeros at $\pi$ is trivially controlled).

1) A 1-D to 2-D Mapping: Furthermore, we also provide a novel mapping of the proposed 1-D filter banks into the 2D quincunx case, preserving all the desirable properties. In particular, there is the following:

1) The perfect reconstruction property is preserved.

2) In the IIR case, all the analysis and synthesis filters remain causal and stable. In the FIR case the linear phase property is preserved.

3) Even though the filter bank is nonseparable, the complexity is that of a separable filter bank, growing linearly with the filter order.

4) The frequency response supports for the filters are the diamond and diamond-complement as desired for the quincunx case [15], [2]. Moreover the filter frequency responses are ensured to be good simply by designing the 1-D filter having a good frequency response. Any desired specifications can be met by designing a 1-D transfer function $\beta(z)$ appropriately as we shall demonstrate.

5) If the 1-D lowpass filter $H_{0}(z)$ has $k$ zeros at $\pi$, then the resulting 2-D lowpass filter will have its $i$ th-order total derivative equal to zero at $(\pi, \pi)$, for $i=0,1, \cdots, k-1$. See Section V for details.

We also provide a design example to show that the mapping can be easily applied to any dilation matrix (i.e., decimation matrix) with determinant 2.

2) Relation to Other Results in the Literature: All the designs proposed in this paper are based on a single class of polyphase matrices to be described in Section V. However, some of the filter banks reported by other researchers are related to our work. In [23], the authors derive a class of biorthogonal linear phase FIR filter bank which turns out to be a special case of our two-channel framework. In the IIR maximally flat halfband case, our solution is different from the traditional IIR Butterworth design and has approximately linear-phase in the passband. In the FIR maximally flat halfband case, the solution agrees with the classical FIR maximally flat design [24]. However, our construction is different from those in [25] and [6] since the analysis filters are factors of maximally flat halfband filters in [25] and [6], whereas our 
analysis filters are themselves maximally flat halfband. The 2-D mapping proposed earlier in [15] and [16] is different from ours because it is known that the earlier mapping will not preserve the PR property in general.

\section{Outline of the Paper and Notations}

Our presentation will go as follows: In the next section, we will derive a framework for the two-channel biorthogonal filter banks. Some properties of such class will be described in detail. In Section III, we will discuss both the IIR and FIR filter banks which are covered in the proposed framework. In Section IV, wavelet basis functions generated from the proposed filter banks will be presented and imposition of zeros at aliasing frequency will be considered. Two new classes of IIR maximally flat solution are given in closed form. In Section V, we will first introduce a novel 2-D mapping for the quincunx case. Some properties of the mapping are discussed. Then both the IIR and FIR cases are considered. Furthermore numerical examples will be provided throughout the discussion to demonstrate the idea.

1) Notations and Definitions: Capital boldfaced letters are used to denote matrices. I represents the identity matrix. The determinant of the matrix $\mathbf{A}$ is denoted by $[\operatorname{det} \mathbf{A}]$. Superscript ${ }^{2-D}$ is used to represent the 2-D function obtained by applying the mapping, for example, $\mathbf{E}^{2-\mathrm{D}}\left(z_{0}, z_{1}\right)$ is obtained by applying the 2-D mapping to $\mathbf{E}(z)$. The $z$-transform of $h(n)$ is represented by $H(z)$. The relation between the filters $\left\{H_{k}(z), F_{k}(z)\right\}$ and the polyphase matrices $\mathbf{E}(z)$ and $\mathbf{R}(z)$ can be described as follows:

$$
\text { and } \quad \begin{aligned}
H_{k}(z) & =E_{k, 0}\left(z^{2}\right)+z^{-1} E_{k, 1}\left(z^{2}\right), \\
F_{k}(z) & =z^{-1} R_{0, k}\left(z^{2}\right)+R_{1, k}\left(z^{2}\right)
\end{aligned}
$$

where $E_{i, j}(z)$ and $R_{i, j}(z)$ are, respectively, the $i j$ th elements of the matrices $\mathbf{E}(z)$ and $\mathbf{R}(z)$. A filter $H_{k}(z)$ is halfband if either one of its polyphase components $E_{k, 0}(z), E_{k, 1}(z)$ is a delay.

\section{A FRAMEWORK FOR 1-D BIORTHOGONAL FILTER BANKS}

Consider Fig. 1, where a two-channel system is shown. In general, $\mathbf{R}(z)=\mathbf{E}^{-1}(z)$ for perfect reconstruction. It is not easy to constrain $[\operatorname{det} \mathbf{E}(z)]$ to be minimum phase for stability of $\mathbf{R}(z)$; therefore, let us make it a delay. An example is

$$
\mathbf{E}(z)=\left(\begin{array}{cc}
z^{-N} & \beta(z) \\
0 & z^{-N^{\prime}}
\end{array}\right)
$$

With this, we obtain

$$
H_{0}(z)=z^{-2 N}+z^{-1} \beta\left(z^{2}\right)
$$

but $H_{1}(z)=z^{-\left(2 N^{\prime}+1\right)}$, which is a delay. Thus, even though $H_{0}(z)$ can be designed to be a good lowpass filter (as we will show), $H_{1}(z)$ is allpass and this is not useful for subband coding applications. We can modify $H_{1}(z)$ without affecting $H_{0}(z)$ by taking the polyphase matrix to be

$$
\begin{aligned}
\mathbf{E}(z) & =\left(\begin{array}{cc}
0.5 & 0 \\
-0.5 \alpha(z) & 1
\end{array}\right)\left(\begin{array}{cc}
z^{-N} & \beta(z) \\
0 & z^{-N^{\prime}}
\end{array}\right) \\
& =\left(\begin{array}{cc}
0.5 z^{-N} & 0.5 \beta(z) \\
-0.5 z^{-N} \alpha(z) & -0.5 \alpha(z) \beta(z)+z^{-N^{\prime}}
\end{array}\right) .
\end{aligned}
$$

Then, we get the following expressions for the analysis filters:

$$
\begin{aligned}
& H_{0}(z)=\frac{\left(z^{-2 N}+z^{-1} \beta\left(z^{2}\right)\right)}{2}, \\
& H_{1}(z)=-\alpha\left(z^{2}\right) H_{0}(z)+z^{-2 N^{\prime}-1} .
\end{aligned}
$$

\section{A. Obtaining Ideal Responses with (4)}

First notice that the filter $H_{0}(z)$ can be made an ideal lowpass filter if $\beta(z)$ has the following magnitude and phase responses:

$$
\begin{aligned}
& \left|\beta\left(e^{j 2 \omega}\right)\right|=1, \quad \forall \omega \\
& \angle \beta\left(e^{j 2 \omega}\right)=\left\{\begin{array}{cl}
(-2 N+1) \omega, & \text { for } \quad \omega \in[0, \pi / 2] \\
(-2 N+1) \omega \pm \pi, & \text { for } \quad \omega \in(\pi / 2, \pi] .
\end{array}\right.
\end{aligned}
$$

From (4), we see that in the high-frequency region, $H_{1}\left(e^{j \omega}\right)$ has unity gain since $\left|H_{0}\left(e^{j \omega}\right)\right|=0$. The function $\alpha(z)$ does not affect $H_{0}(z)$ and can be freely chosen to shape the response of $H_{1}(z)$. It should be chosen such that in the low frequency region, $\alpha\left(z^{2}\right) H_{0}(z)$ cancels with $z^{-2 N^{\prime}-1}$. For exact magnitude cancellation, $\left|\alpha\left(e^{j \omega}\right)\right|$ must be unity. Since $H_{0}(z)$ is linear phase, it is necessary that $\alpha(z)$ has linear phase in the low-frequency region. Comparing these two requirements and the conditions in (5), we realize that $\beta(z)$ is a suitable candidate for $\alpha(z)$. Indeed, if $N^{\prime}=2 N-$ $1, H_{1}(z)$ is an ideal highpass filter. In this case, we have an ideal filter bank, and the polyphase matrix $\mathbf{E}(z)$ in Fig. 1(b) is

$$
\begin{aligned}
\mathbf{E}(z) & =\left(\begin{array}{cc}
0.5 & 0 \\
-0.5 \beta(z) & 1
\end{array}\right)\left(\begin{array}{cc}
z^{-N} & \beta(z) \\
0 & z^{-2 N+1}
\end{array}\right) . \\
& =\left(\begin{array}{cc}
0.5 z^{-N} & 0.5 \beta(z) \\
-0.5 z^{-N} \beta(z) & -0.5 \beta^{2}(z)+z^{-2 N+1}
\end{array}\right) .
\end{aligned}
$$

With this, we get the following expressions for the analysis filters, which we will repeatedly use in this paper.

$$
\begin{aligned}
& H_{0}(z)=\frac{\left(z^{-2 N}+z^{-1} \beta\left(z^{2}\right)\right)}{2}, \\
& H_{1}(z)=-\beta\left(z^{2}\right) H_{0}(z)+z^{-4 N+1} .
\end{aligned}
$$

The perfect reconstruction can be achieved by choosing $\mathbf{R}(z)$ in Fig. 1(b) to be

$$
\begin{aligned}
\mathbf{R}(z) & =\left(\begin{array}{cc}
z^{-2 N+1} & -\beta(z) \\
0 & z^{-N}
\end{array}\right)\left(\begin{array}{cc}
1 & 0 \\
0.5 \beta(z) & 0.5
\end{array}\right) \\
& =\left(\begin{array}{cc}
z^{-2 N+1}-0.5 \beta^{2}(z) & -0.5 \beta(z) \\
0.5 z^{-N} \beta(z) & 0.5 z^{-N}
\end{array}\right) .
\end{aligned}
$$

The corresponding synthesis filters can be verified to have the following form:

$$
F_{0}(z)=-H_{1}(-z), \quad F_{1}(z)=H_{0}(-z)
$$

This choice of synthesis filters in (9) ensures that $\left\{F_{0}(z)\right.$, $\left.F_{1}(z)\right\}$ is a lowpass/highpass pair if $\left\{H_{0}(z), H_{1}(z)\right\}$ is a lowpass/highpass pair. From (6) and (8), we have the implementation of the filter bank shown in Fig. 2. The structure is similar to a ladder network structure [26]. 


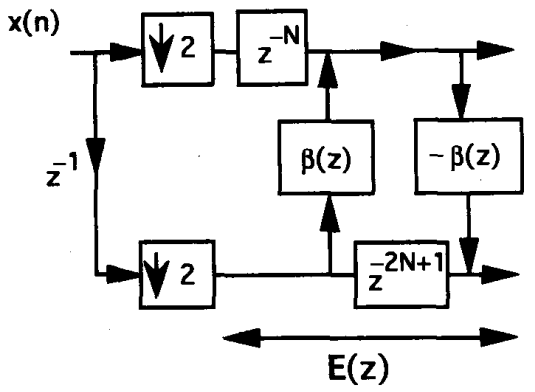

Fig. 2. Implementation of the proposed biorthogonal filter bank.

Remarks: Of course, the $\alpha(z)$ in (3) can be taken as functions different from $\beta(z)$, as in the case of [20], [21], [23]. This will provide more freedom in the design. However, by taking them to be the same, the biorthogonal systems can have some additional useful properties. Therefore, we will only consider the case when $\alpha(z)=\beta(z)$.

\section{B. Two Useful Approximations of (5)}

The ideal choice of $\beta(z)$ as in (5) requires infinite complexity. Therefore, we have to design $\beta(z)$ to approximate the conditions in (5). However the approximation will not change the perfect reconstruction property because $\mathbf{E}(z)$ in (6) and $\mathbf{R}(z)$ in (8) satisfy $\mathbf{R}(z) \mathbf{E}(z)=0.5 z^{-3 N+1} \mathbf{I}$, regardless of the choice of $\beta(z)$. Fig. 2 shows that the frequency responses of all the analysis and synthesis filters depend on one single function $\beta(z)$ only. The frequency selectivity of all four filters depends on how well $\beta(z)$ approximates conditions (5). This makes the design procedure simple. In the next section, we will provide two simple but useful approximations which correspond to the following two cases:

1) Stable IIR case: Here, $\beta(z)$ is chosen to be a causal stable allpass function so that (5a) is met exactly. We design the phase response of the allpass filter so that (5b) is approximately satisfied. This leads to a biorthogonal system with causal stable IIR analysis and synthesis filters.

2) Linear phase FIR case: To satisfy the condition (5b), $\beta(z)$ can be chosen as a Type 2 linear phase function [2] (filter with a symmetric impulse response of even length). The magnitude response of $\beta(z)$ is optimized to be as close to unity as possible so that (5a) is well approximated. This leads to a linear phase biorthogonal system.

\section{Additional Properties of the Filter Banks Designed as Above}

In Section I, we have outlined some properties. Properties 1-4 mentioned at the beginning of Section I-B are clear from the above discussion and Property 5 will be discussed in the Section IV. In addition to these five properties, we have the following:

1) Double halfband property: In all the previous constructions of two-channel PR filter banks, $H_{0}(z) F_{0}(z)$ is a halfband filter, where $H_{0}(z)$ is not necessary halfband

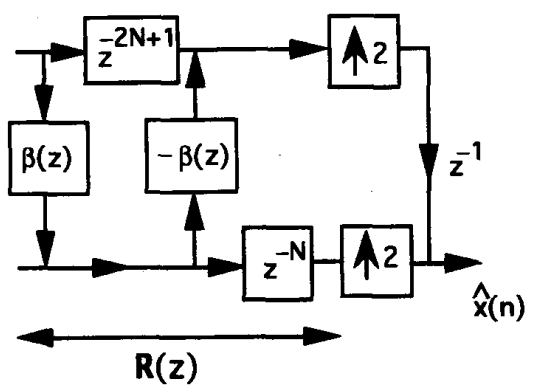

but a factor of a halfband filter. However in our construction above, one can verify that not only the product $H_{0}(z) F_{0}(z)$ but also the filter $H_{0}(z)$ is halfband.

2) Poles of filters: In the IIR case, notice from Fig. 2 that there is no feedback loop in both the analysis and synthesis ends in the ladder network. Therefore, the filters have the same poles as those of $\beta\left(z^{2}\right)$ and stability depends solely on the allpass function $\beta(z)$. Moreover in the IIR case if the allpass filter $\beta(z)$ is implemented by using the robust lattice structure [2], the filter bank is stable even when it is realized with finite wordlength.

3) Robustness to round off noise: The ladder structure shown in Fig. 2 is similar to the structure considered in [26]. By using the same reasoning in [26], it can be verified that the round off noise in the analysis end is compensated by that in the synthesis end. Combining this with the structurally PR property, we conclude that the implementation in Fig. 2 preserves PR even when all the coefficients are quantized to a finite precision and all the intermediate results are rounded off. However, if the subband signals are quantized (which is usually the case), this property is lost.

4) Zeros of the filters: We can verify that $F_{0}(z)$ and $H_{1}(z)$ in (9) and (7) can, respectively, be rewritten as

$$
\begin{aligned}
& F_{0}(z)=\left(2 z^{-2 N+1}-\beta\left(z^{2}\right)\right) H_{0}(z), \\
& H_{1}(z)=\left(2 z^{-2 N+1}+\beta\left(z^{2}\right)\right) F_{1}(z) .
\end{aligned}
$$

These factorizations give the filter bank an interesting structure shown in Fig. 3. From (10), it is clear that if $\beta(z)$ is FIR, the zeros of $H_{0}(z)$ are also zeros of $F_{0}(z)$. Even when $\beta(z)$ is an irreducible IIR transfer function, this is true since $H_{0}(z)$ is in the form of (7) and the zeros of denominator of $\beta\left(z^{2}\right)$ cannot cancel the zeros of $H_{0}(z)$. Moreover, if $\left|\beta\left(e^{j \omega}\right)\right|<2$, both $F_{0}\left(e^{j \omega}\right)$ and $H_{0}\left(e^{j \omega}\right)$ have the same set of zeros on the unit circle. The same is true for the pair of $H_{1}(z)$ and $F_{1}(z)$. In particular, if $H_{0}(z)$ has $r$ zeros at $z=-1$, this implies that $F_{0}(z)$ has no fewer than $r$ zeros at the same point. This property is important in the generation of wavelets since for biorthogonal wavelets, we need both of the analysis and the synthesis wavelets to be regular. By increasing the number of zeros of $H_{0}(z)$ at $z=-1$, our construction ensures that $F_{0}(z)$ has at least the same number of zeros at $z=-1$. This is the property which 


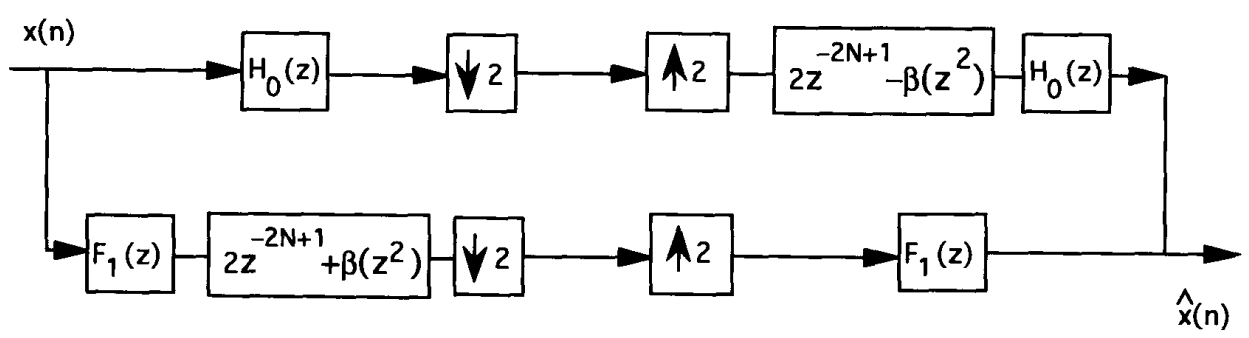

Fig. 3. Redrawing of Fig. 2, where $H_{0}(\xi)=0.5\left(z^{-2 N}+z^{-1} 3\left(z^{2}\right)\right)$ and $F_{1}(\xi)=H_{0}(-z)$.

does not appear in the previously existing constructions of biorthogonal filter banks.

5) Ripple sizes of the filters: Since $H_{0}(z)$ is a halfband filter and $H_{0}(z)+F_{1}(z)=z^{-2 N}$, we have the following relationship between the passband ripple $\delta_{p}$ and the stopband ripple $\delta_{s}$ :

$$
\delta_{p}\left(H_{0}\right)=\delta_{s}\left(H_{0}\right)=\delta_{p}\left(F_{1}\right)=\delta_{s}\left(F_{1}\right) .
$$

Moreover, by using $(10)$ and the fact that $\beta\left(z^{2}\right) \approx$ $-z^{-2 N+1}$ in the high frequency region, we get

$$
\delta_{s}\left(F_{0}\right) \approx 3 \delta_{s}\left(H_{0}\right), \quad \delta_{s}\left(H_{1}\right) \approx 3 \delta_{s}\left(F_{1}\right)
$$

$(20 \log 3 \approx 9.5 \mathrm{~dB})$. This property ensures that by designing $H_{0}(z)$ to have sufficiently high stopband attenuation, we can ensure that all the other three filters will also have good frequency selectivity.

6) Complexity: From Fig. 2, it is very clear that the analysis and synthesis banks have the same complexity. Assume that $\beta(z)$ has order $N$. For the IIR case, by using the one multiplier lattice structure for allpass function [2], we need approximately $2 N$ multiplications, $6 N$ additions, and $5 N$ delays. For the FIR case, by exploiting the symmetry, we need approximately $N$ multiplications, $2 \mathrm{~N}$ additions and $3.5 \mathrm{~N}$ delays. All the operations are at a lower rate. Therefore, the analysis (or synthesis) bank requires $N$ and $0.5 N$ multiplications per input sample for the IIR and FIR case, respectively.

7) Near linear phase in the IIR cases: From (7), since in the passband the magnitude response of $H_{0}(z)$ is approximately one, the transfer function $\beta\left(z^{2}\right) \approx z^{-2 N+1}$. Therefore, $H_{0}(z)$ has approximately linear phase in the passband. Similar argument is true for $H_{1}(z)$.

\section{Design Procedures for the Two}

Classes of BIORTHOGONAL FILTER BANKS

In this section, we will discuss the two cases of the approximations of (5) given in the last section. Simple design procedures will be given for both cases.

\section{A. Causal Stable IIR Biorthogonal Filter Banks}

In this section, $\beta(z)$ in (6)-(9) is taken to be the causal stable real allpass function

$$
A_{N_{1}}(z)=\frac{\sum_{k=0}^{N_{1}} a_{N_{1}, N_{1}-k} z^{-k}}{\sum_{k=0}^{N_{1}} a_{N_{1}, k} z^{-k}}
$$

where $a_{N_{1}, 0}=1$ and $a_{N_{1}, k}$ are real. In this case, $H_{0}(z)$ is a sum of a delay and an allpass function. See (7). It is an IIR halfband filter and has been studied by some researchers [27], [28]. $H_{0}(z)$ can be made lowpass with large stopband attenuation and small passband ripples by designing the phase response of the allpass function to approximate (5b) [29].

1) Choice of $N_{1}$ : From the monotone decreasing phase property [2] of a causal stable allpass function, we know that the phase of $A_{N_{1}}\left(z^{2}\right)$ spans a range of $4 N_{1} \pi$ when $\omega$ spans a range of $2 \pi$, but from $(5 b), \beta\left(z^{2}\right)$ spans a range of $4 N \pi$ or $4(N-1) \pi$. To make the range spanned by both of the functions equal, we set $N_{1}=N$ or $N-1$ and this results in two classes of causal stable IIR filter banks. Since the derivation and properties of both of the classes are very similar, in the rest of the paper, we consider only the case $N_{\ell}=N$ (we will point out at those places where the second class has a different property). With this choice, the analysis filters can be written as

$$
\begin{aligned}
& H_{0}(z)=\frac{\left(z^{-2 N}+z^{-1} A_{N}\left(z^{2}\right)\right)}{2} \\
& H_{1}(z)=-A_{N}\left(z^{2}\right) H_{0}(z)+z^{-4 N+1} .
\end{aligned}
$$

The relationship between the synthesis and analysis filters is the same as (9).

\section{2) Additional Properties of the Above IIR Filter Banks:}

1) Preservation of zero at aliasing frequency: Substituting $z=-1$ into the expression of $H_{0}(z)$ in (14), we find that $H_{0}(z)$ always has a zero at $z=-1$, independent of the coefficients $a_{N, k}$. In particular, the zero is preserved even when all $a_{N, k}$ are quantized coarsely. This means that one zero at $z=-1$ is structurally imposed. This is important in the generation of wavelet bases since one zero at $z=-1$ is a necessary condition for the existence of the wavelet functions [6]. Note also that $H_{1}(z)$ will always have a structurally imposed zero at $z=1$.

2) Low sensitivity: Since there exists low sensitivity lattice structure for allpass function [2], the filters have low passband sensitivity. Since the halfband property of $H_{0}(z)$ is structurally imposed, it has low stopband sensitivity as well.

3) Bump in the transition band: Substituting $\omega=\pi / 2$ into the expression for $H_{1}\left(e^{j \omega}\right)$ and $F_{0}\left(e^{j \omega}\right)$ and using the fact that $A_{N}(-1)=(-1)^{N}$, we find that $\left|H_{1}\left(e^{j \omega}\right)\right|=$ $\left|F_{0}\left(e^{j \omega}\right)\right|=\sqrt{2.5}$ at $\omega=\pi / 2$, independent of the allpass function $A_{N}(z)$. This means that $\left|H_{1}\left(e^{j \omega}\right)\right|$ and $\left|F_{0}\left(e^{j \omega}\right)\right|$ always have a bump of approximately $4 \mathrm{~dB}$ 


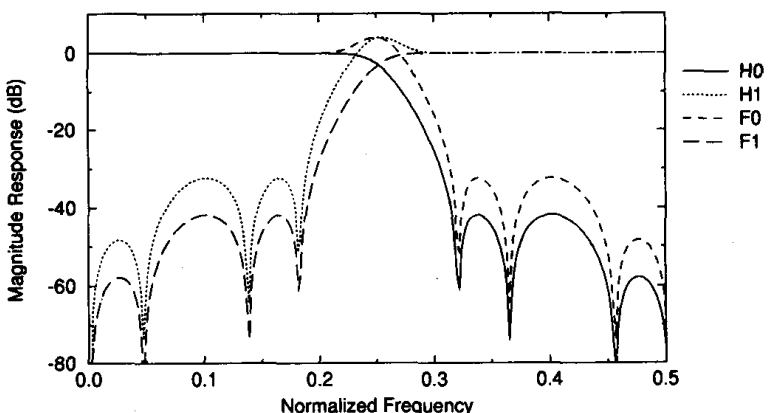

(a)

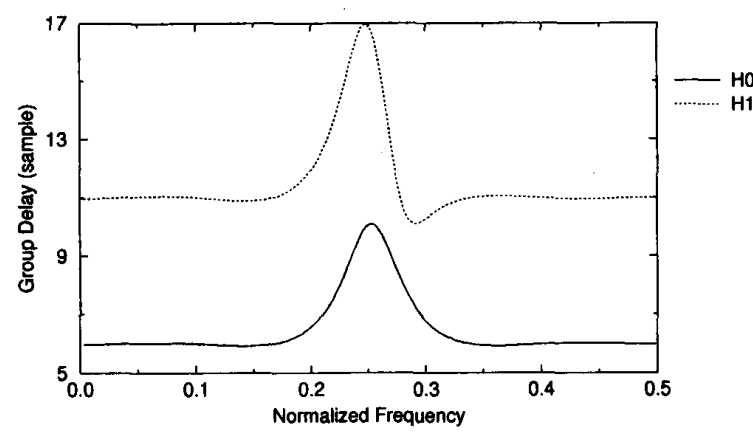

(b)

Fig. 4. (Example 3.1) Frequency responses of the causal stable IIR filter bank: (a) Magnitude responses of the analysis and synthesis filters; (b) group delays of $H_{0}(z)$ and $H_{1}(z)$.

at $\omega=\pi / 2$, no matter how we design $A_{N}(z)$. The width but not amplitude of the bump can be reduced by increasing the complexity of $A_{N}(z)$.

Example 3.I-I-D Causal Stable IIR Filter Banks: In this example, $N=3$. Therefore, $A_{N}(z)$ is a third-order allpass function. The filter bank has very low complexity: To implement the analysis (or synthesis) bank, we need only three multiplications per input sample! By using the eigenfilter approach for allpass functions [29], we optimize the coefficients $a_{k}$ such that maximum attenuation in the stopband of $H_{0}(z)$ is achieved. The coefficients are obtained as $a_{3,1}=0.473$, $a_{3,2}=-0.094$, and $a_{3,3}=0.025$. For the filter $H_{0}(z)$, the passband edge $\omega_{p}=0.4 \pi$ and the stopband edge $\omega_{s}=0.6 \pi$. The stopband attenuation $\delta_{s}\left(H_{0}\right)=41.9 \mathrm{~dB}$. The magnitude responses of the all four filters are shown in Fig. 4(a). From the plots, relations of ripple sizes in (11) and (12) can be verified and it is clear that $H_{0}(z)$ and $F_{0}(z)$ have the same set of zeros on the unit circle. The bump of approximately $4 \mathrm{~dB}$ around $\pi / 2$ is clearly seen. The group delay for $H_{0}(z)$ and $H_{1}(z)$ is shown in Fig. 4(b). The filters are approximately linear phase in the passband and the stopband.

\section{B. Linear Phase FIR Biorthogonal Filter Banks}

In the linear phase FIR case, since $H_{0}(z)$ is a linear phase halfband filter, it can be designed by employing the trick developed in [30], viz., by taking $\beta(z)$ in (5)-(8) to be a Type 2 filter [2] which has a symmetric impulse response of length

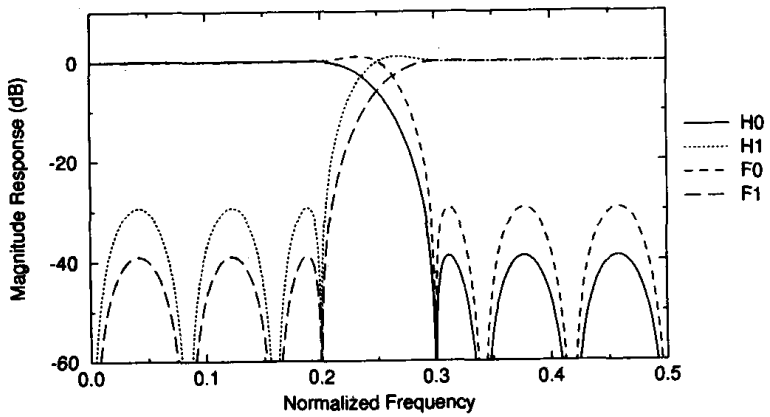

Fig. 5. (Example 3.2) Magnitude responses of the linear phase FIR filter bank.

$2 N_{1}$. In this case, the number of multiplications required to implement $\beta(z)$ is $N_{1}$, the same as the $N_{1}$ th-order allpass function $A_{N_{1}}(z)$ in (13). More precisely, let $\beta(z)$ have the following form:

$$
V(z)=\sum_{k=1}^{N_{1}} v_{k} \times\left(z^{-N_{1}+k}+z^{-N_{1}-k+1}\right)
$$

where the coefficients $v_{k}$ satisfy

$$
\sum_{k=1}^{N_{1}} v_{k}=0.5
$$

so that $V\left(e^{j 0}\right)=1$ and $H_{0}\left(e^{j 0}\right)=1$. It is well-known that a Type 2 linear phase filter always has a zero at $z=-1$. In order to satisfy the condition (5b) exactly, it can be verified that $N_{1}$ should be equal to $N$. By employing the trick in [30], the coefficients $v_{k}$ can be optimized such that the amplitude response of $V\left(e^{j \omega}\right)$ is as close to unity as possible. In this case, the analysis filters are:

$$
\begin{aligned}
& H_{0}(z)=\frac{\left(z^{-2 N}+z^{-1} V\left(z^{2}\right)\right)}{2}, \\
& H_{1}(z)=-V\left(z^{2}\right) H_{0}(z)+z^{-4 N+1} .
\end{aligned}
$$

Example 3.2-I-D Linear Phase FIR Filter Banks: $N=6$. To implement the analysis bank, we need six multiplications per input sample, double the number in Example 3.1. The Type 2 linear phase function $V(z)$ is designed by using McClellan"Park algorithm. The coefficients are obtained as $v_{1}=0.630, v_{2}=-0.193, v_{3}=0.0972, v_{4}=-0.0526, v_{5}=$ 0.0272 , and $v_{6}=-0.0144$. For the filter $H_{0}(z)$, the passband edge $\omega_{p}=0.4 \pi$ and the stopband edge $\omega_{s}=0.6 \pi$, same condition as Example 3.1. The stopband attenuation $\delta_{s}\left(H_{0}\right)=$ $39.2 \mathrm{~dB}$ and $\delta_{s}\left(H_{1}\right)=30 \mathrm{~dB}$. The magnitude responses of all four filters are shown in Fig. 5. The relations of ripple sizes in (11) and (12) can be verified.

For comparison, we will consider Johnston's design with nearly the same specifications. The Johnston's filter $24 \mathrm{C}$ in (see appendix 7.1 of [31]) has $\delta_{s}=30 \mathrm{~dB}$ and $\omega_{s}=0.586 \pi$. For Johnston's filter 32-D, $\delta_{s}=38 \mathrm{~dB}$ and $\omega_{s}=0.586 \pi$. To implement the analysis bank, we need respectively 12 multiplications and 16 multiplications per input sample for the above two cases. Thus, as compared to six multiplications in our filter bank, the Johnston's design has more complexity 
than our design. Moreover, there is reconstruction error $(0.1$ $\mathrm{dB}$ for $24 \mathrm{C}$ and $0.025 \mathrm{~dB}$ for $32 \mathrm{D}$ ) in Johnston's filter bank.

\section{IMPOSITION OF MULTIPLE ZeROS AT $\pi$}

The relation between continuous-time wavelet and discretetime perfect reconstruction filter bank is well known. A way to construct the scaling and wavelet functions from the filter coefficients was first given by Daubechies in [6]. Starting from the impulse response coefficients $h_{0}(n)$ and $h_{1}(n)$, a pair of continuous-time functions $\phi_{H_{0}}(x)$ and $\psi_{H_{1}}(x)$ are constructed such that they satisfy:

$$
\begin{aligned}
& \phi_{H_{0}}(x)=\sum_{n=0}^{\infty} h_{0}(n) \phi_{H_{0}}(2 x-n), \\
& \psi_{H_{1}}(x)=\sum_{n=0}^{\infty} h_{1}(n) \phi_{H_{0}}(2 x-n) .
\end{aligned}
$$

Here, $\phi_{H_{0}}(x)$ and $\psi_{H_{1}}(x)$ are respectively called the analysis scaling and wavelet functions. For the synthesis end, we can write similar expressions for the synthesis scaling and wavelet functions, $\phi_{F_{0}}(x)$ and $\psi_{F_{1}}(x)$. The conditions for the existence of such limit functions were given in [6]. It is always desirable to have smooth or "regular" limit functions. It was shown that in order to achieve limit functions of high regularity, we need to have a sufficient number of zeros at the aliasing frequency $\pi$. Therefore in the rest of this section, we will show how to impose zeros at $\pi$ for the proposed filter banks.

\section{A. Causal Stable IIR Wavelet Bases}

For the purpose of achieving regularity, we impose multiple zeros of $H_{0}(z)$ at $\pi$. Since the denominator does not provide any zeros, we consider only the numerator of $H_{0}(z)$. Except for a delay, the numerator of $H_{0}(z)$ can be written in terms of $a_{N, k}$ as follows:

$$
P_{R}(\omega)=\sum_{k=0}^{N} a_{N, k} \cos (2 k-1 / 2) \omega .
$$

To obtain $r$ zeros at $z=-1$, we set

$$
\left.P_{R}^{(i)}(\pi) \triangleq \frac{d^{(i)}}{d \omega^{(i)}} P_{R}(\omega)\right|_{\omega=\pi}=0, \text { for } i=1,2, \cdots, r-1
$$

Note that when $i$ is even, $P_{R}^{(i)}(\pi)$ is always equal to zero. This proves that $P_{R}(\omega)$ always has an odd number of zeros at $\omega=\pi$. Therefore, we can write $r=2 r_{0}+1$. In this case, we obtain a set of $r_{0}$ linear constraints as follows:

$$
\sum_{k=0}^{N} a_{N, k}(1-4 k)^{2 i-1}=0, \text { for } i=1,2, \cdots, r_{0} .
$$

The set of linear constraints in (21) can be satisfied exactly in the optimization of the phase response of the allpass function $A_{N}(z)$ by using the efficient eigenfilter approach [29], [32].

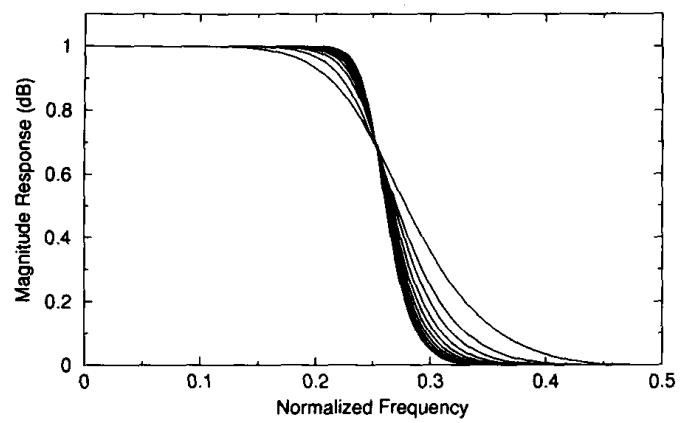

Fig. 6. Magnitude responses of the IIR maximally flat filters of the form $0.5\left[z^{-2 N}+z^{-1} A_{N}\left(z^{2}\right)\right]$, where $A_{N}(z)$ is a $N$ th-order all pass function, for $N=1,2, \cdots, 10$.

1) Maximally Flat IIR Wavelets: To obtain a maximally flat solution, i.e., maximum possible number of zeros at $\pi$ consistent with the constraint that $H_{0}(z)=0.5\left(z^{-2 N}+z^{-1} A_{N}\left(z^{2}\right)\right)$, we set $r_{0}$ in (21) as large as possible. However, if $r_{0} \geq N+1$, then we can list the first $(N+1)$ linear constraints given by (21) as follows:

$$
\begin{gathered}
\underbrace{\left(\begin{array}{cccc}
1 & 1 & \cdots & 1 \\
x_{0}^{2} & x_{1}^{2} & \cdots & x_{N}^{2} \\
\vdots & \vdots & \ddots & \vdots \\
x_{0}^{2 N} & x_{1}^{2 N} & \cdots & x_{N}^{2 N}
\end{array}\right)}_{\text {Vandermonde }}\left(\begin{array}{llll}
x_{0} & & & \\
& x_{1} & & \\
& & \ddots & \\
& & & x_{N}
\end{array}\right) \\
\times\left(\begin{array}{c}
a_{N, 0} \\
a_{N, 1} \\
\vdots \\
a_{N, N}
\end{array}\right)=\left(\begin{array}{c}
0 \\
0 \\
\vdots \\
0
\end{array}\right)
\end{gathered}
$$

where $x_{k}=1-4 k$. Since all the $x_{k}$ are nonzero and distinct, the two matrices on the left hand side are nonsingular and hence invertible. We get $\left[a_{N, 0} a_{N, 1} \cdots a_{N, N}\right]^{T}=0$ which violates the requirement that $a_{N, 0}=1$. This proves under the constraint that $H_{0}(z)=0.5\left[z^{-2 N}+z^{-1} A_{N}\left(z^{2}\right)\right]$, the filter $H_{0}(z)$ can have at most $2 N+1$ zeros at $\pi$. Indeed we can show that the maximally flat IIR filter has exactly $2 N+1$ zeros at $\pi$. To see this, we set $r_{0}=N$ and rewrite the set of $N$ linear equations given by (21) as follows:

$$
\begin{gathered}
\left(\begin{array}{cccc}
1 & 1 & \cdots & 1 \\
x_{1}^{2} & x_{2}^{2} & \cdots & x_{N}^{2} \\
\vdots & \vdots & \ddots & \vdots \\
x_{1}^{2 N-2} & x_{2}^{2 N-2} & \cdots & x_{N}^{2 N-2}
\end{array}\right)\left(\begin{array}{cccc}
x_{1} & & & \\
& x_{2} & & \\
& & \ddots & \\
& & & x_{N}
\end{array}\right) \\
\times\left(\begin{array}{c}
a_{N, 1} \\
a_{N, 2} \\
\vdots \\
a_{N, N}
\end{array}\right)=-\left(\begin{array}{c}
1 \\
1 \\
\vdots \\
1
\end{array}\right)
\end{gathered}
$$

where $x_{k}=1-4 k$ and the fact that $a_{N, 0}=1$ has been imposed. These equations fully determine $A_{N}(z)$ (hence all the filters) and there is no further parameter to be optimized numerically. As the matrices are invertible, the solution for $a_{N, k}$ always exists and it is unique. Furthermore, it is shown in 


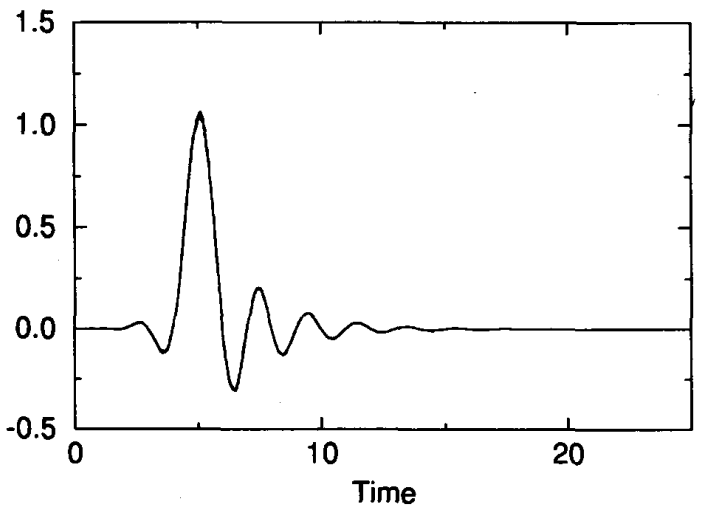

(a)

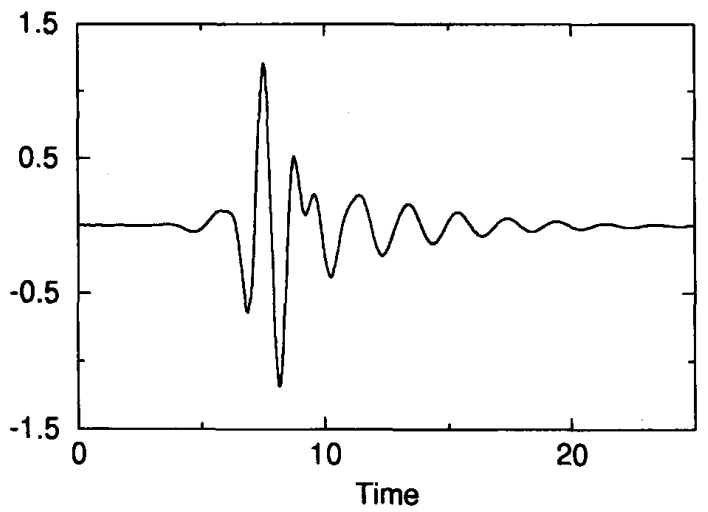

(b)

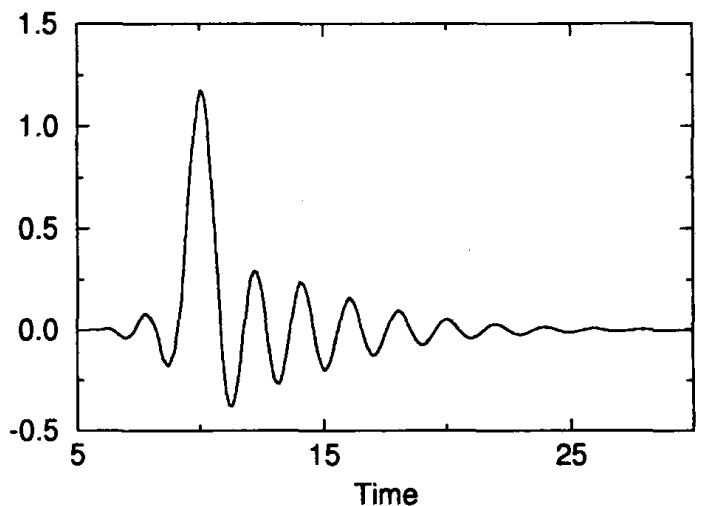

(c)

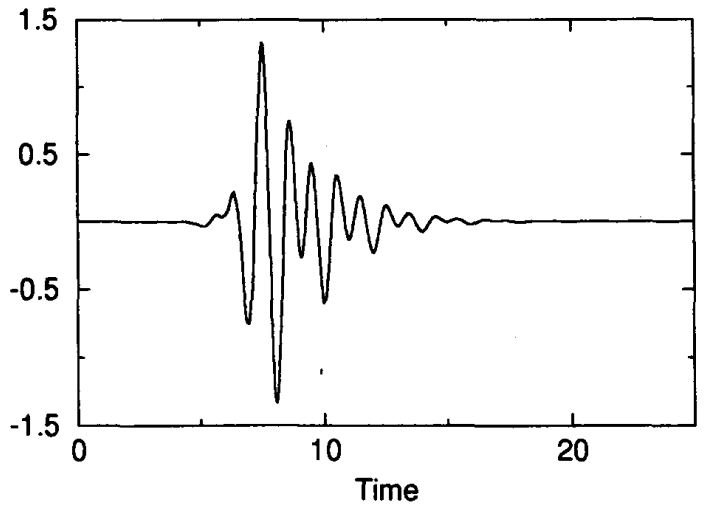

(d)

Fig. 7. (Example 4.1 i)) The limit functions generated by using the IIR filter bank in Example $3.1\left(H_{0}(z)\right.$ has one zero at $\pi$ ): (a) Analysis scaling function; (b) analysis wavelet function; (c) synthesis scaling function; (d) synthesis wavelet function.

Appendix A that $a_{N, k}$ has the following closed-form solution:

$$
a_{N, k}=\frac{(-1)^{k-1}}{2 k-1}\left(\begin{array}{l}
N \\
k
\end{array}\right) \prod_{i=1}^{N} \frac{(2 i-1)}{(2 k+2 i-1)}, \quad 0 \leq k \leq N
$$

where $\left(\begin{array}{l}N \\ k\end{array}\right)=\frac{N !}{(N-\bar{k}) ! k !}$. The frequency responses of $H_{0}(z)$ corresponding to $N=1,2, \cdots, 10$ are shown in Fig. 6. Note that although these filters have a numerator of degree $4 N-$ 1 (excluding the trivial delay factor), they have only $2 N+1$ zeros at $z=-1$. This implies that some of the zeros are not at $z=-1$ for $N>1$ and therefore these IIR maximally flat filters are different from the Butterworth halfband filters. Moreover they have nearly linear phase in the passband, as justified at the end of Section II and demonstrated in Fig. 4(b). For the case of $N=1$, one can verify that the solution is a third-order Butterworth filter.

Remarks:

1) If the function $\beta(z)$ is taken as $(N-1)$ th-order allpass filter (i.e., $N_{1}=N-1$ ), then we will get a second class of causal stable IIR wavelet. In this case, under the constraint that $H_{0}(z)=0.5\left[z^{-2 N}+z^{-1} A_{N-1}\left(z^{2}\right)\right]$, the process of imposition of zeros at $\pi$ is very similar to the derivation above. The maximally flat IIR filter $H_{0}(z)$ of this second class will have $2 N-1$ zeros at $\pi$.
The closed form solution for $a_{N-1, k}$ is given as

$$
\begin{aligned}
a_{N-1, k} & =\frac{(-1)^{k}}{2 k+1}\left(\begin{array}{c}
N-1 \\
k
\end{array}\right) \prod_{i=1}^{N-1} \frac{(2 i+1)}{(2 k+2 i+1)}, \\
1 & \leq k \leq N-1
\end{aligned}
$$

and $a_{N-1,0}=1$.

2) Notice that for a perfect reconstruction system, if we interchange the analysis and synthesis filters, the perfect reconstruction property is retained. In many applications such as coding, compression, storage and approximation, the regularity of the synthesis functions is more important [17]. Thus we can choose the wavelet with higher regularity among $\psi_{H_{0}}(x)$ and $\psi_{F_{0}}(x)$ as the synthesis wavelet.

Example 4.1: We generate the limit functions, $\phi_{H_{0}}, \psi_{H_{1}}, \phi_{F_{0}}$, and $\psi_{F_{1}}$, corresponding to the filter bank in Fig. 1(a). To generate the analysis/synthesis scaling and wavelet functions, we use the cascade algorithm in [6] for eight iterations. We consider the following two cases:

i) No linear constraint is set, $H_{0}(z)$ has only one zero at $\pi$. The analysis and synthesis filters are the same as those in Example 3.1. For the analysis bank, the scaling and 


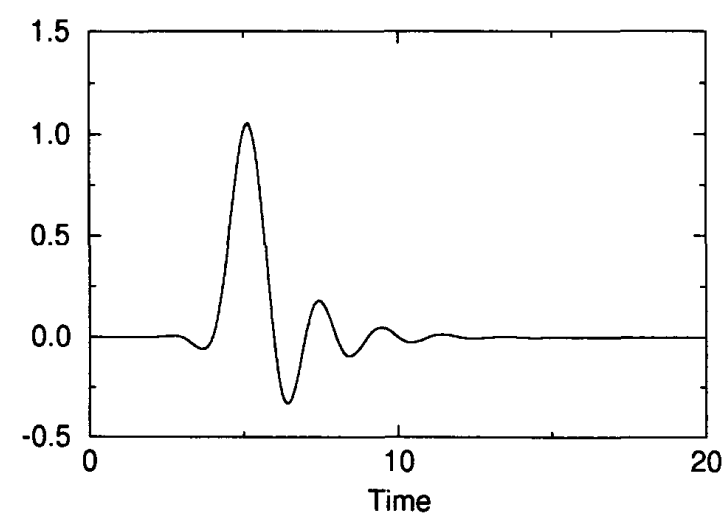

(a)

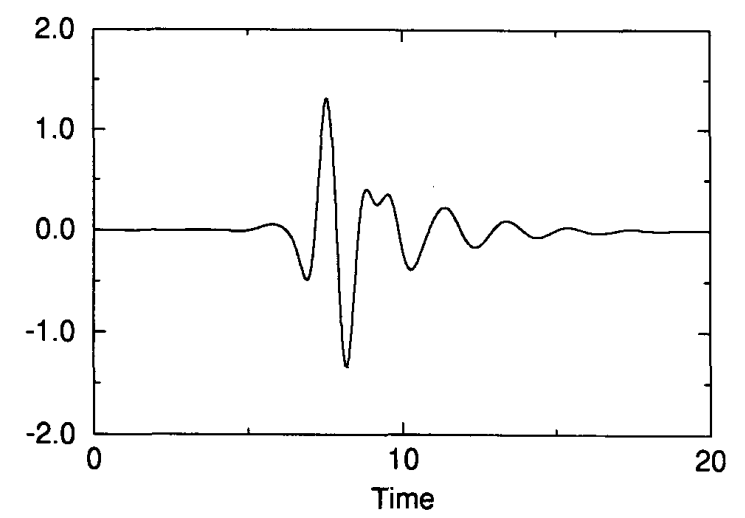

(d)

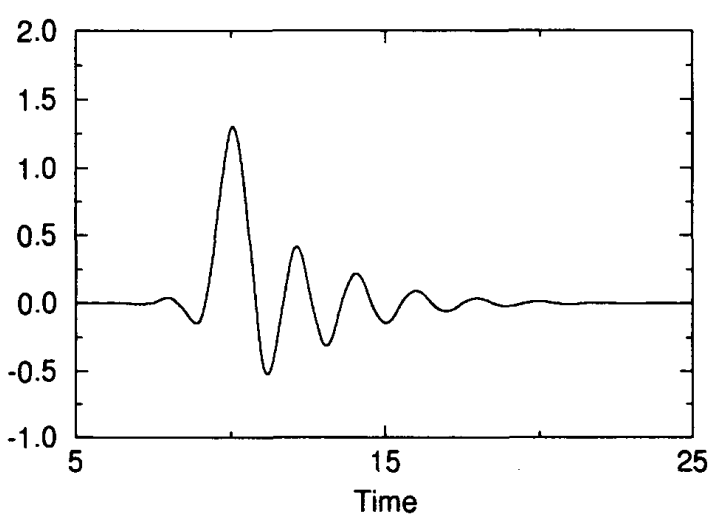

(c)

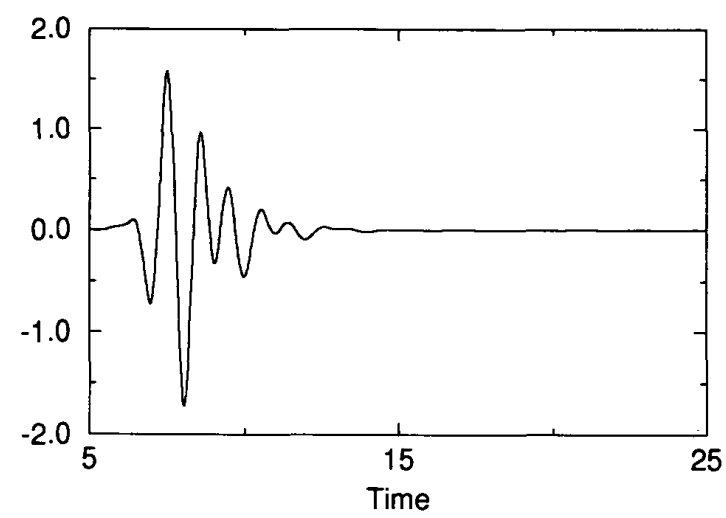

(e)

Fig. 8. (Example 4.1 ii)) Limit functions generated by using the IIR maximally flat filter bank $\left(H_{0}(Z)\right.$ has seven zeros at $\left.\pi\right)$ : (a) Analysis scaling function; (b) analysis wavelet function; (c) synthesis scaling function; (d) synthesis wavelet function.

wavelet functions, $\phi_{H_{0}}$ and $\psi_{H_{1}}$, are respectively shown in Fig. 7(a) and (b). The scaling and wavelet functions corresponding to the synthesis bank, $\phi_{F_{0}}$ and $\psi_{F_{1}}$, are shown in Fig. 7(c) and (d).

ii) As a comparison, we also generate the scaling and wavelet functions corresponding to the IIR maximally flat filters $\left(\phi_{\max }\right.$ and $\psi_{\max }$ ) for $N=3$. In this case, the filter $H_{0}(z)$ has seven zeros at $\pi$. The limit functions are shown in Fig. 8. For a better comparison on smoothness, in Fig. 9 we show a zoom-in for Figs. 7(a) and 8(a). We see that the limit functions in Fig. 8 are more regular than the functions shown in Fig. 7.

\section{B. Linear-Phase FIR Wavelet Bases}

To impose multiple zeros at $\pi$ for the linear phase FIR case, the procedure is very similar to that given above. Another set of linear constraints can be obtained and incorporated in the procedure of optimization. It can be verified that for this case, $H_{0}(z)$ always has an even number of zeros at $\pi$.

1) Maximally Flat Linear Phase FIR Wavelets: The FIR maximally flat filters have been studied by a number of researchers [24], [33], [6], [25]. In [6] and [25], a maximally flat halfband FIR filter is used to construct compactly

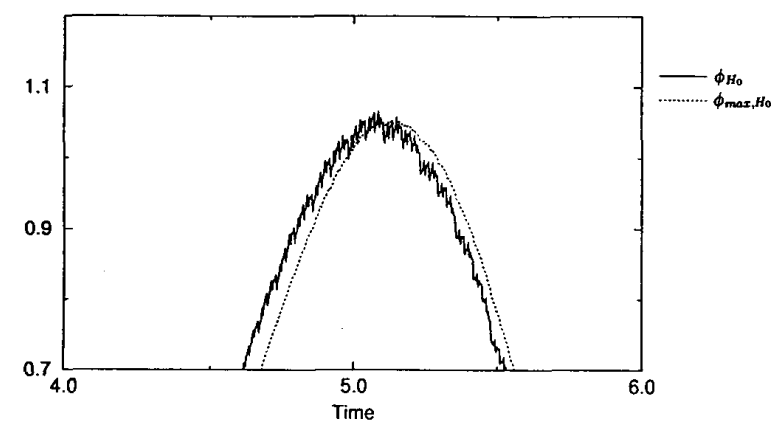

Fig. 9. Zoom-in for Figs. 7(a) and 8(a) demonstrating the improved "regularity" obtained by imposing zeros at $\pi$.

supported maximally flat wavelets. In our linear phase FIR filter bank, if all the freedom is used to impose zeros at $\pi$, we will arrive at the same solution as that in [6], [25]. The closed form solution for FIR maximally flat halfband filters was in [33], [25] as

$$
v_{k}=\frac{(-1)^{N+k-1} \prod_{i=0}^{2 N}(N+1 / 2-i)}{2(N-k) !(N-1+k) !(2 k-1)}
$$




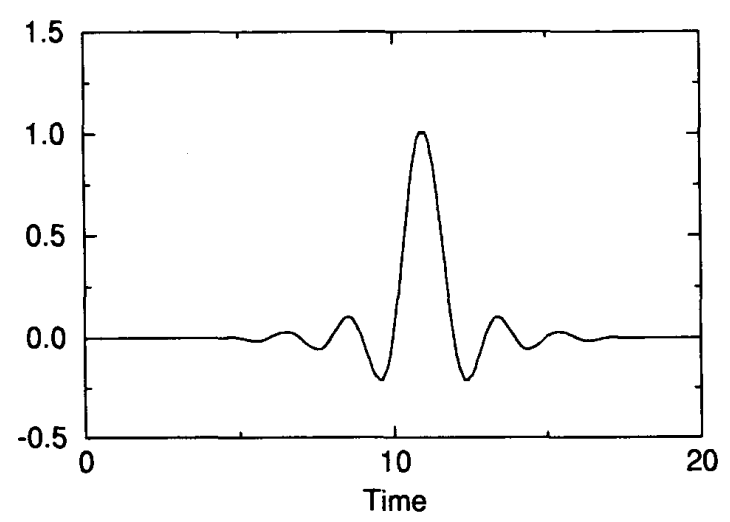

(a)

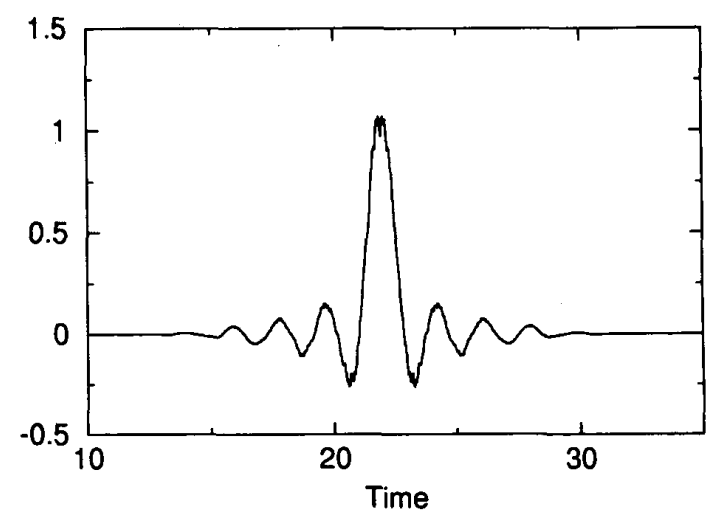

(b)

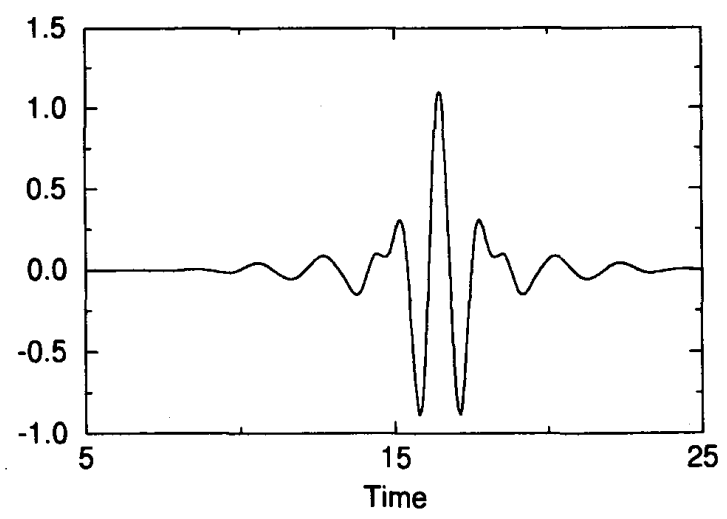

(c)

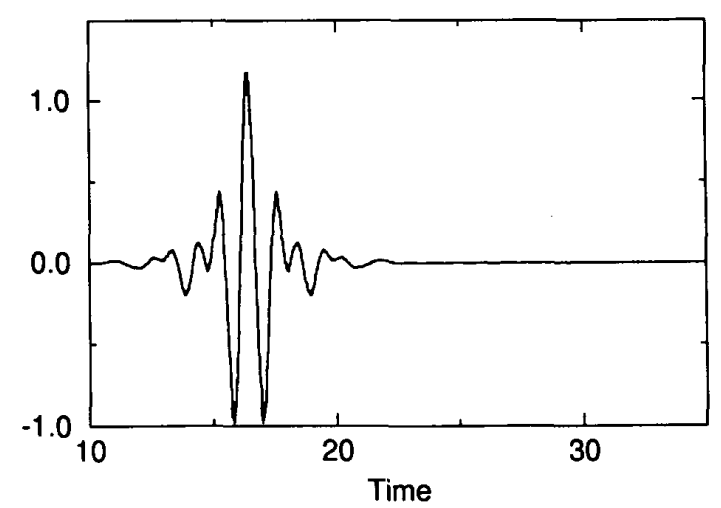

(d)

Fig. 10. (Example 4.2 i)) Symmetric limit functions generated by using the FIR filter bank in Example $3.2\left(H_{0}(z)\right.$ has two zeros at $\pi$ ): (a) Analysis scaling function; (b) analysis wavelet function; (c) synthesis scaling function; (d) synthesis wavelet function.

2) Differences Between Our Construction and that in [25] and [6]: In [25], $H_{0}(z)$ is taken to be a factor of a maximally flat halfband filter. In [6], power spectral factorization is considered. However, in our linear phase structure, $H_{0}(z)$ is taken to be this halfband filter itself, and not a factor. Since the $H_{0}(z)$ constructed in [6] is a power spectral factor of the $H_{0}(z)$ in our structure, our linear phase scaling function $\phi_{\mathrm{LP}}(x)$ is related to that constructed by Daubechies in [6], $\phi_{D}(x)$ as:

$$
\phi_{\mathrm{LP}}(x)=\phi_{D}(x) * \phi_{D}^{*}(-x)
$$

where $*$ denotes convolution and $\phi_{D}^{*}$ denotes the complex conjugate of $\phi_{D}$. From (27), it is clear that the regularity of $\phi_{\mathrm{LP}}(x)$ is twice that of $\phi_{D}(x)$. However the order (and the number of zeros at $\pi$ ) of $H_{0}(z)$ in our construction is twice that of $H_{0}(z)$ in the construction in [6]. Comparing the complexity, both of the constructions have approximately the same number of multiplications (because in our construction, linear phase property can be exploited).

Example 4.2: In this example, we construct the limit functions corresponding to the filter bank in Fig. 1(a) for the linear phase FIR case. The cascade algorithm is used for eight iterations. We consider two cases: i) First, $H_{0}(z)$ is designed such that no linear constraint other than (16) is satisfied, therefore it has two zeros at $\pi$. The analysis and synthesis filters are the same as those in Example 3.2. The limit functions $\left(\phi_{H_{0}}, \psi_{H_{1}}, \phi_{F_{0}}\right.$ and $\psi_{F_{1}}$ ) are respectively shown in Fig. 10(a)-(d).

ii) For a comparison, we show the limit functions of the maximum flat case $\left(\phi_{\max }\right.$ and $\psi_{\max }$ ) for $N=6$. In this case, $H_{0}(z)$ has twelve zeros at $\pi$. The plots are shown in Fig. 11. It can be verified that the limit functions in Fig. 11 are smoother than those in Fig. 10.

\section{MAPPING INTO 2-D QUINCUNX PERFECT RECONSTRUCTION FILTER BANKS}

In this section, we will generalize the 1-D framework discussed in Section II to the 2-D case. We will focus on the quincunx subsampling case which has the subsampling lattice shown in Fig. 12. Notice that the dilation matrix has determinant 2 . The corresponding maximally decimated filter bank has only two channels. Furthermore it represents the simplest nonseparable subsampling lattice.

In the 2-D case, we know that the desired passband supports of the filters depend not only on the lattice but also on the choice of dilation matrix $\mathbf{M}$ [12]. In the rest of this section, 


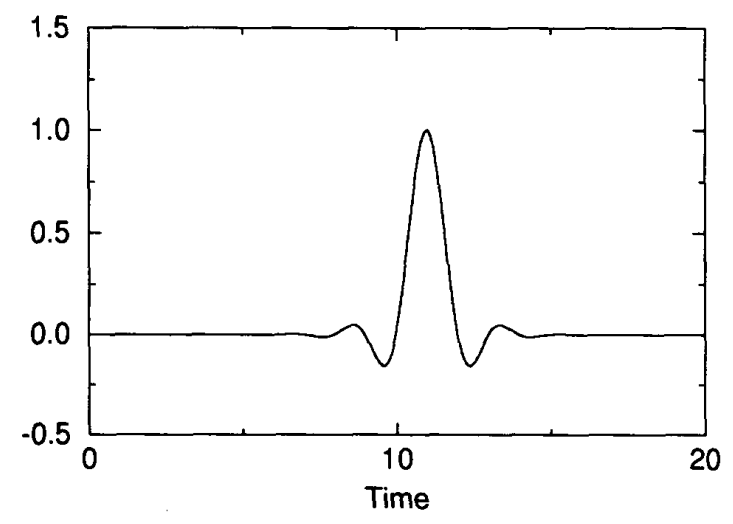

(a)

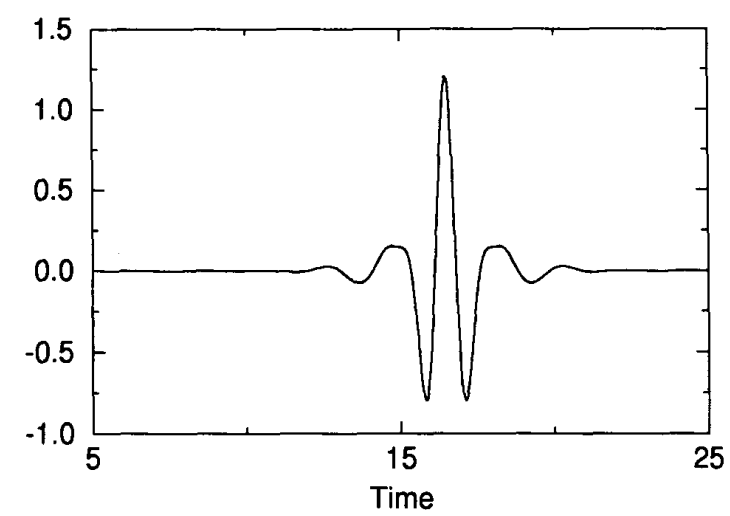

(b)

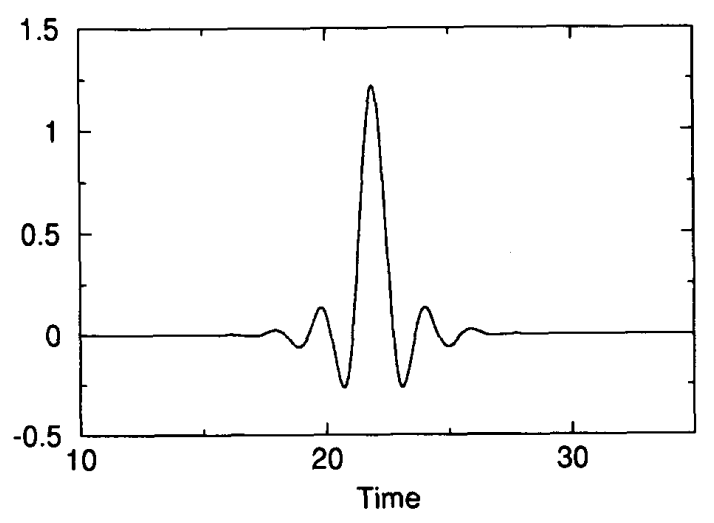

(c)

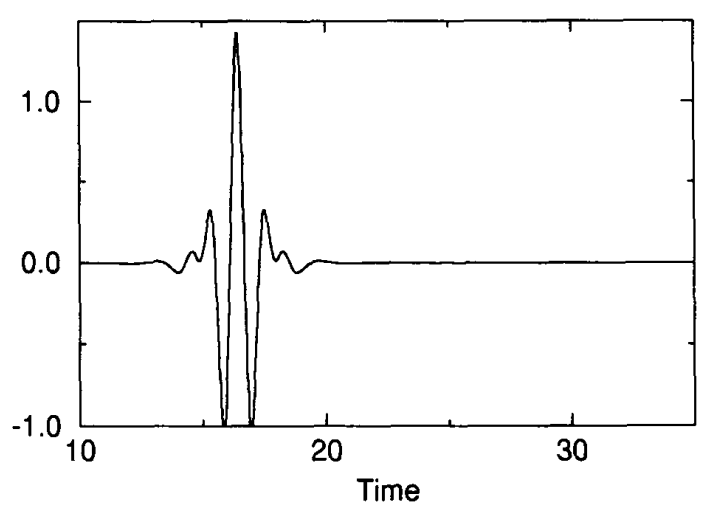

(d)

Fig. 11. (Example 4.2 ii)) Symmetric limit functions generated by using the FIR maximally flat filter bank $\left(H_{0}(\approx)\right.$ has 12 zeros at $\pi$ ): (a) Analysis scaling function; (b) analysis wavelet function; (c) synthesis scaling function; (d) synthesis wavelet function.

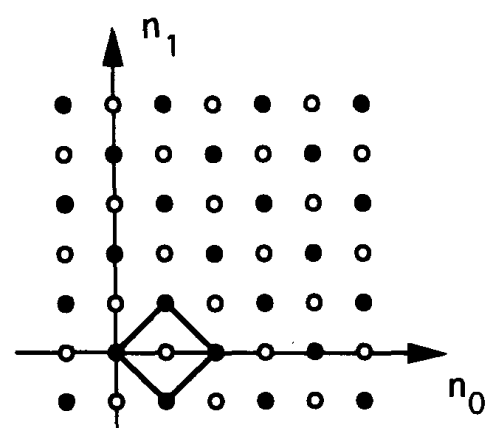

Fig. 12. Quincunx subsampling lattice.

we will consider

$$
\mathbf{M}=\left(\begin{array}{cc}
1 & 1 \\
1 & -1
\end{array}\right)
$$

The coset vectors are, respectively

$$
\mathbf{k}_{0}=\left(\begin{array}{l}
0 \\
0
\end{array}\right), \quad \mathbf{k}_{1}=\left(\begin{array}{l}
1 \\
0
\end{array}\right) .
$$

With this $\mathbf{M}$, the ideal supports for alias free decimation, $\operatorname{SPD}\left(\pi \mathbf{M}^{-T}\right)[2$, ch. 12] is shown in Fig. 13, where the diamond and diamond-complement, $\Omega_{0}$ and $\Omega_{1}$, correspond to the low frequency and high frequency regions respectively. One can verify that $\mathbf{M}$ defined in (28) has its eigenvalues $\lambda_{i}$ equal to $\pm \sqrt{2}$ and $\mathbf{M}^{2}=2 \mathbf{I}$. It has a dilation in both the directions. Therefore, $\mathbf{M}$ satisfies the conditions for a wellbehaved matrix defined in [19]. Given the dilation matrix $\mathbf{M}$ as in (28) and the coset vectors in (29), the simple delay chain system and the noble identities are shown in Fig. 14(a) and (b), respectively.

Although the discussion in this paper is mainly on the quincunx subsampling case with the dilation matrix $\mathbf{M}$ and the coset vectors $\mathbf{k}_{i}$ defined above, we will provide a design example in the last section to show that the method discussed in this section can be easily generalized to any 2-D system with decimation matrix $\mathbf{M}$ having $[\operatorname{det} \mathbf{M}]=2$.

\section{A. A 1-D to 2-D Mapping}

In this section, we will first give a 2-D mapping and then apply the mapping to the framework developed in Section II. Given any 1-D biorthogonal systems with the polyphase matrices of the form in (6) and (8), we will use the following transformation on the polyphase components: 


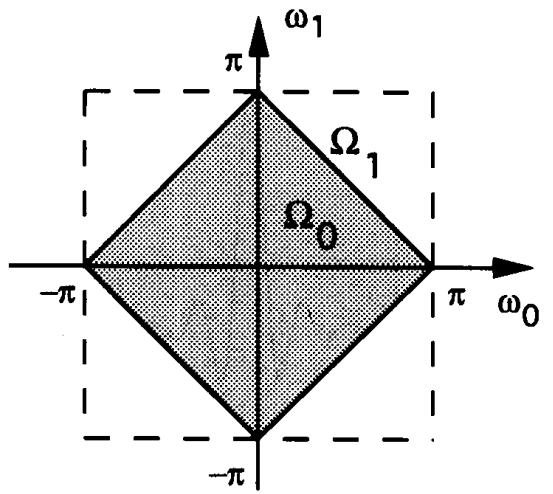

Fig. 13. Ideal supports for alias-free decimation in quincunx case.

i) First replace the 1-D transfer function $\beta(z)$ with the separable 2-D transfer function $\beta\left(z_{0}\right) \beta\left(z_{1}\right)$.

ii) Replace all the remaining 1-D delay $z^{-1}$ with the 2-D delay $z_{0}^{-1} z_{1}^{-1}$.

This results in nonseparable analysis and synthesis filters as we will see. Under this transformation, the polyphase matrices $\mathbf{E}^{2-\mathrm{D}}\left(z_{0}, z_{1}\right)$ and $\mathbf{R}^{2-\mathrm{D}}\left(z_{0}, z_{1}\right)$ of the 2-D system can be written, respectively, as (30) and (31), which appear at the bottom of the page. From (30) and (31), we have the implementation of the 2-D perfect reconstruction filter bank as Fig. 15. By using the noble identities in Fig. 14, we can write the analysis and synthesis filters as

$$
\begin{aligned}
H_{0}\left(z_{0}, z_{1}\right) & =\frac{z_{0}^{-2 N}+z_{0}^{-1} \beta\left(z_{0} z_{1}^{-1}\right) \beta\left(z_{0} z_{1}\right)}{2} \\
H_{1}\left(z_{0}, z_{1}\right) & =-\beta\left(z_{0} z_{1}^{-1}\right) \beta\left(z_{0} z_{1}\right) H_{0}\left(z_{0}, z_{1}\right)+z_{0}^{-4 N+1},(32 \mathrm{a}) \\
F_{0}\left(z_{0}, z_{1}\right) & =-H_{1}\left(-z_{0},-z_{1}\right), \quad F_{1}\left(z_{0}, z_{1}\right)=H_{0}\left(-z_{0},-z_{1}\right) .
\end{aligned}
$$

1) Comparison of the Above Transformation with Those in [15]-[17]: McClellan's transformation is used in [17] to obtain a FIR maximally flat halfband filter. The transformation proposed in this paper differs from McClellan's transformation in the sense that the former operates on the polyphase components while the latter operates directly on the filter. In

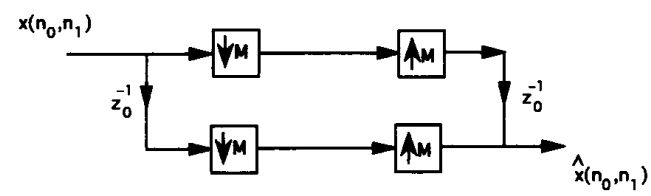

(a)

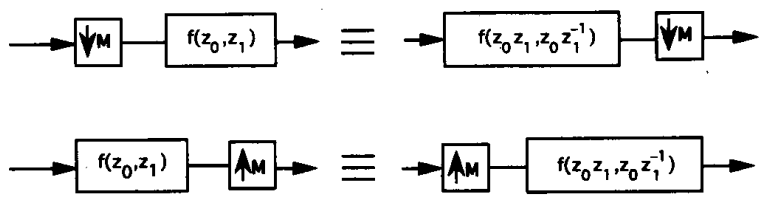

(b)

Fig. 14. Some details for the quincunx decimator: (a) Delay chain perfect reconstruction system; (b) noble identities.

[15], [16], the authors obtain a 2-D filter bank from 1-D by employing the following transformation:

$$
\mathbf{E}_{i, j}^{2-\mathrm{D}}\left(z_{0}, z_{1}\right)=\mathbf{E}_{i, j}\left(z_{0}\right) \mathbf{E}_{i, j}\left(z_{1}\right)
$$

where $\mathbf{E}_{i, j}$ is the $(i, j)$ th element of $\mathbf{E}$. It is clear that in our transformation $\mathbf{E}_{1,1}^{2-D}\left(z_{0}, z_{1}\right) \neq \mathbf{E}_{1,1}\left(z_{0}\right) \mathbf{E}_{1,1}\left(z_{1}\right)$. Therefore, our mapping is different from that in (33).

2) Properties of the proposed 2-D mapping: Properties 1-5 in Section II continue to hold after minor modifications to suit the 2-D context. In addition, the 2-D filter bank satisfies the following properties:

1) Double Halfband Property: It is easy to see that $H_{0}\left(z_{0}, z_{1}\right)$ satisfies $H_{0}\left(z_{0}, z_{1}\right)+H_{0}\left(-z_{0},-z_{1}\right)=z_{0}^{-2 N}$ and $H_{0}\left(z_{0}, z_{1}\right) F_{0}\left(z_{0}, z_{1}\right)$ satisfies a similar property. This is the extension of the 1-D double halfband property in the 2-D quincunx case.

2) Stability of the 2-D Analysis and Synthesis Filters: If the 1-D transfer function $\beta(z)$. is causal then so are the functions $\beta\left(z_{0}\right) \beta\left(z_{1}\right)$ in Fig. 15. That is $\beta\left(z_{0}\right) \beta\left(z_{1}\right)$ is a first-quadrant filter (the impulse response is zero unless $n_{0} \geq 0$ and $\left.n_{1} \geq 0\right)$. If $\beta(z)$ is BIBO stable, then so is $\beta\left(z_{0}\right) \beta\left(z_{1}\right)$ so that the polyphase matrix in Fig. 15 is also BIBO stable. Since the analysis filters are obtained from this stable structure, these filters are guaranteed to be BIBO stable. However we see

$$
\begin{aligned}
\mathbf{E}^{2-\mathrm{D}}\left(z_{0}, z_{1}\right)= & \left(\begin{array}{cc}
0.5 & 0 \\
-0.5 \beta\left(z_{0}\right) \beta\left(z_{1}\right) & 1
\end{array}\right)\left(\begin{array}{cc}
\left(z_{0} z_{1}\right)^{-N} & \beta\left(z_{0}\right) \beta\left(z_{1}\right) \\
0 & \left(z_{0} z_{1}\right)^{-2 N+1}
\end{array}\right) \\
= & \left(\begin{array}{cc}
0.5\left(z_{0} z_{1}\right)^{-N} & 0.5 \beta\left(z_{0}\right) \beta\left(z_{1}\right) \\
-0.5\left(z_{0} z_{1}\right)^{-N} \beta\left(z_{0}\right) \beta\left(z_{1}\right) & -0.5 \beta^{2}\left(z_{0}\right) \beta^{2}\left(z_{1}\right)+\left(z_{0} z_{1}\right)^{-2 N+1}
\end{array}\right) \\
\mathbf{R}^{2-\mathrm{D}}\left(z_{0}, z_{1}\right)= & \left(\begin{array}{cc}
\left(z_{0} z_{1}\right)^{-2 N+1} & -\beta\left(z_{0}\right) \beta\left(z_{1}\right) \\
0 & \left(z_{0} z_{1}\right)^{-N}
\end{array}\right)\left(\begin{array}{cc}
1 & 0 \\
0.5 \beta\left(z_{0}\right) \beta\left(z_{1}\right) & 0.5
\end{array}\right) \\
= & \left(\begin{array}{cc}
\left(z_{0} z_{1}\right)^{-2 N+1}-0.5 \beta^{2}\left(z_{0}\right) \beta^{2}\left(z_{1}\right) & -0.5 \beta\left(z_{0}\right) \beta\left(z_{1}\right) \\
0.5\left(z_{0} z_{1}\right)^{-N} \beta\left(z_{0}\right) \beta\left(z_{1}\right) & 0.5\left(z_{0} z_{1}\right)^{-N}
\end{array}\right)
\end{aligned}
$$




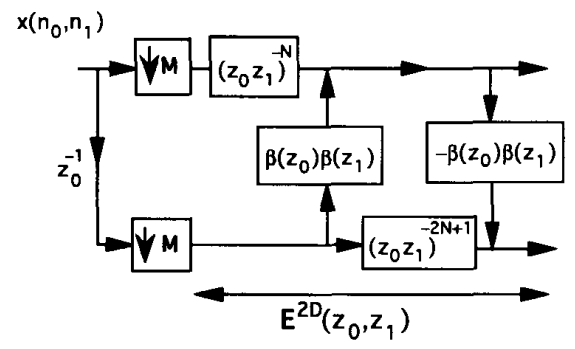

Fig. 15. 2-D biorthogonal filter bank obtained from Fig. 2 by mapping.

that the term $\beta\left(z_{0} z_{1}^{-1}\right)$ has entered the expressions for the analysis filters because of the noble identities, see Fig. 14(b). It can be shown that this violates the condition for the so-called first-quadrant stability [35, p. 166]. This is explained by the fact that the analysis filters are not first-quadrant filters, even though BIBO stable. This is consistent with the observation that the quincunx decimator $\mathbf{M}$ in (28) has the negative entry -1 . Indeed, the expression $y(\mathbf{n})=x(\mathbf{M n})$ means $y\left(n_{0}, n_{1}\right)=x\left(n_{0}+n_{1}, n_{0}-n_{1}\right)$ so that there is a timereversal operation buried in the decimation process. The same remarks apply for the synthesis filters, that is the 2-D synthesis filters $F_{0}\left(z_{0}, z_{1}\right)$ and $F_{1}\left(z_{0}, z_{1}\right)$ are BIBO stable even though they are not first-quadrant filters.

3) Perfect reconstruction is preserved.

4) If the 1-D lowpass filter $H_{0}(z)$ has $k$ zeros at $\pi$, then the frequency response of $H_{0}\left(e^{j \omega_{0}}, e^{j \omega_{1}}\right)$ can be written as

$$
\begin{aligned}
H_{0}\left(e^{j \omega_{0}}, e^{j \omega_{1}}\right)= & \left(1+e^{-j \frac{\omega_{0}+\omega_{1}}{2}}\right)^{k} P_{1}\left(\omega_{0}, \omega_{1}\right) \\
& -\left(1+e^{-j \frac{\omega_{0}-\omega_{1}+2 \pi}{2}}\right)^{k} P_{2}\left(\omega_{0}, \omega_{1}\right)
\end{aligned}
$$

where $\left|P_{1}(\pi, \pi)\right|$ and $\left|P_{2}(\pi, \pi)\right|$ are finite quantities. The proof of (34) is given in Appendix B. Notice that both of the factors $\left[1+e^{-0.5 j\left(\omega_{0}+\omega_{1}\right)}\right]$ and $\left[1+e^{-0.5 j\left(\omega_{0}-\omega_{1}+2 \pi\right)}\right]$ are zero at $(\pi, \pi)$. Furthermore one can verify that all the mixed partial derivatives satisfy

$$
\left.\frac{\partial^{i+l}}{\partial \omega_{0}^{i} \partial \omega_{1}^{l}} H_{0}\left(e^{j \omega_{0}}, e^{j \omega_{1}}\right)\right|_{(\pi, \pi)}=0, \quad \text { for } \quad i+l<k \text {. }
$$

From (35), we conclude that the total derivatives [36]

$$
\begin{aligned}
d^{n} H_{0}(\pi, \pi)= & \sum_{i=0}^{n}\left(\begin{array}{c}
n \\
i
\end{array}\right) d \omega_{0}^{i} d \omega_{1}^{n-i} \frac{\partial^{n}}{\partial \omega_{0}^{i} \partial \omega_{1}^{n-i}} \\
& \times H_{0}(\pi, \pi)=0, \quad \text { for } n<k .
\end{aligned}
$$

According to [34], (36) is a necessary condition for the regularity of 2-D wavelet. The necessary and sufficient condition is still unknown.

5) In the FIR case the linear phase property of the analysis and synthesis filters is preserved.

6) In the IIR case, the 2-D analysis and synthesis filters have a line of zeros in the frequency plane at $\omega_{0}=0$ or at $\omega_{0}=\pi$.

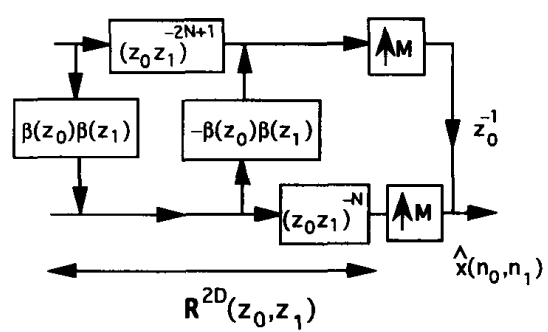

Proof: Substituting $z_{0}=-1$ into the expression for $H_{0}\left(z_{0}, z_{1}\right)$ in (32a) and using the fact that $\beta\left(z_{0} z_{1}\right)$ is allpass, one immediately finds that $H_{0}\left(-1, z_{1}\right)=$ $0, \forall z_{1}$. Since $F_{0}\left(z_{0}, z_{1}\right)$ contains $H_{0}\left(z_{0}, z_{1}\right)$ as a factor, $F_{0}\left(-1, z_{1}\right)=0$. Similarly, we can prove that $F_{1}\left(1, z_{1}\right)=H_{1}\left(1, z_{1}\right)=0, \forall z_{1}$.

7) The lowpass/highpass characteristics of the frequency responses of the filters are preserved.

Proof: Assume that $\beta(z)$ satisfies the ideal conditions in (5). Then we have

$\beta\left(z_{0} z_{1}\right)=\left\{\begin{aligned}\left(z_{0} z_{1}\right)^{\frac{-2 N+1}{2}}, & \text { for } \frac{\omega_{0}+\omega_{1}}{2} \in[0, \pi / 2] ; \\ -\left(z_{0} z_{1}\right)^{\frac{-2 N+1}{2}}, & \text { for } \frac{\omega_{0}+\omega_{1}}{2} \in(\pi / 2, \pi] .\end{aligned}\right.$

$\beta\left(z_{0} z_{1}^{-1}\right)= \begin{cases}\left(z_{0} z_{1}^{-1}\right)^{\frac{-2 N+1}{2}}, & \text { for } \frac{\omega_{0}-\omega_{1}}{2} \in[0, \pi / 2] ; \\ -\left(z_{0} z_{1}^{-1}\right)^{\frac{-2 N+1}{2}}, & \text { for } \frac{\omega_{0}-\omega_{1}}{2} \in(\pi / 2, \pi] .\end{cases}$

By using the above equations, we find that $\beta\left(z_{0} z_{1}\right) \beta\left(z_{0} z_{1}^{-1}\right)$ is equal to $z_{0}^{-2 N+1}$ when $\left(\omega_{0}, \omega_{1}\right) \in \Omega_{0}$ and equal to $-z_{0}^{-2 N+1}$ when $\left(\omega_{0}, \omega_{1}\right) \in \Omega_{1}$. This proves that $H_{0}\left(z_{0}, z_{1}\right)$ has the ideal diamond support $\Omega_{0}$. Similarly it can be shown that $H_{1}\left(z_{0}, z_{1}\right)$ will have the support of ideal diamond-complement. Thus when the conditions in (5) are well-approximated by the 1-D transfer function $\beta(z)$, the response of the 2-D filters will be good.

3) Comments on the Complexity: Though the 2-D analysis and synthesis filters are nonseparable, it is clear from the expressions for the polyphase matrices that the complexity is comparable to that of a separable filter bank. More precisely, it is equal to twice the complexity of the 1-D transfer function $\beta(z)$.

\section{B. 2-D Nonseparable Filter Banks}

In this section, we will give two numerical examples to demonstrate the mapping proposed above. We separately apply the 2-D mapping to the filter banks in Example 3.1 (IIR) and Example 3.2 (FIR), respectively.

Example 5.1-2-D IIR Filter Banks: In this example, we transform the 1-D filter bank in Example 3.1 into the 2-D case by using above mapping. Since $N=3$, the allpass function $A_{3}(z)$ needs only three multiplications. Since the complexity of the 2-D analysis (or synthesis) bank is equal to twice that of $A_{3}(z)$, we need only six multiplications per input pixel to implement the analysis (synthesis) bank. The responses of 


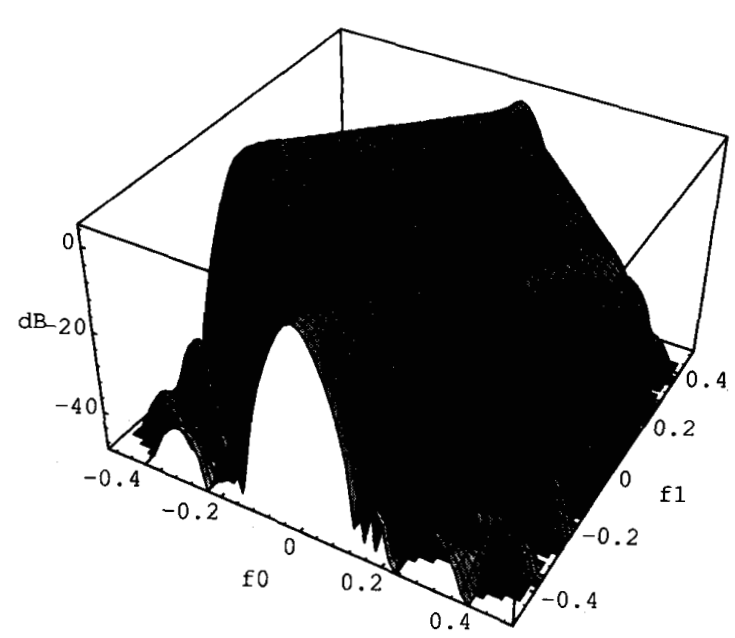

(a)

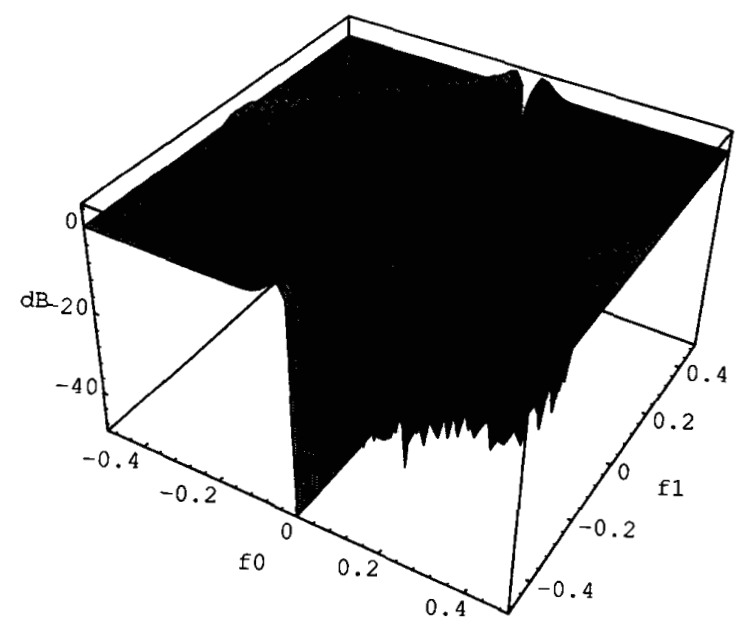

(b)

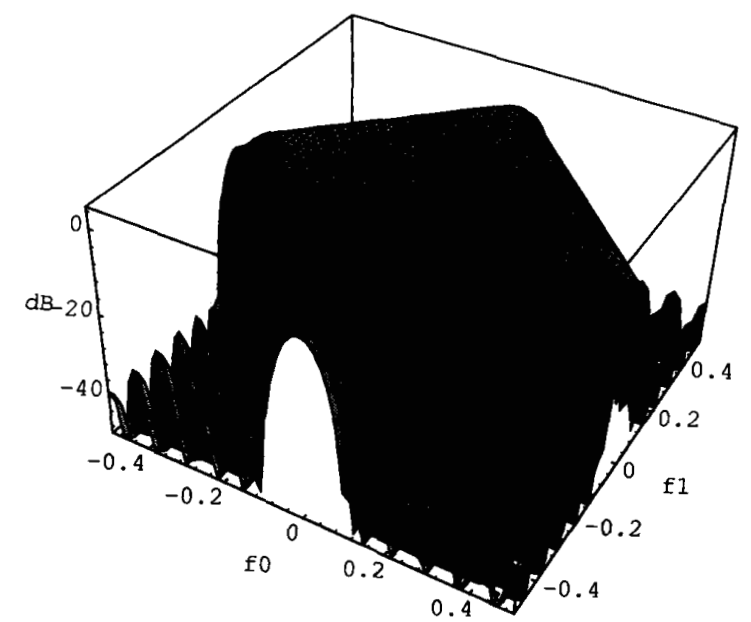

(a)

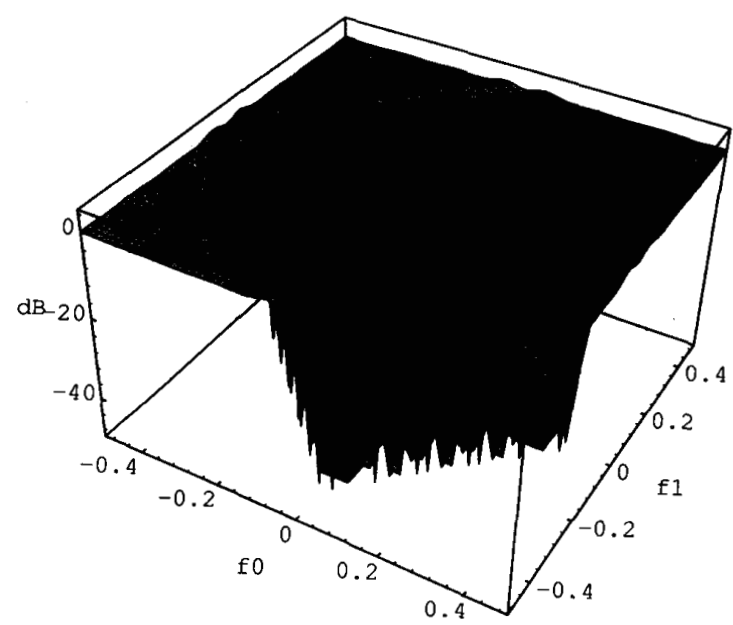

(b)

Fig. 16. (Example 5.1) Magnitude responses of the perfect reconstruction IIR analysis bank: (a) $H_{0}\left(z_{0}, z_{1}\right)$; (b) $H_{1}\left(z_{0}, z_{1}\right)$. The normalized frequency $f_{i}=w_{i} / 2 \pi$.

$H_{0}\left(z_{0}, z_{1}\right)$ and $H_{1}\left(z_{0}, z_{1}\right)$ are shown in Fig. 16(a) and (b), respectively. The supports of the two filters are diamond and diamond-complement, respectively as desired. The stopband attenuation $\delta_{s}\left(H_{0}\right) \approx 42 \mathrm{~dB}$ and $\delta_{s}\left(H_{1}\right) \approx 32 \mathrm{~dB}$. Again, we see that $H_{1}$ is about $10 \mathrm{~dB}$ worse than $H_{0}$ in the stopband. The line of zero of $H_{1}$ at $\omega_{0}=0$ is clearly seen in Fig. 16(b).

Example 5.2-2-D FIR Filter Banks: In this example, the 1-D filter bank in Example 3.2 is transformed into the 2-D case. To implement the 2-D analysis (or synthesis) bank, we need 12 multiplications per input pixel. The magnitude responses of $H_{0}\left(z_{0}, z_{1}\right)$ and $H_{1}\left(z_{0}, z_{1}\right)$ are shown in Fig. 17(a)-(b), respectively. The stopband attenuation $\delta_{s}\left(H_{0}\right) \approx 40$ and $\delta_{s}\left(H_{1}\right) \approx 30 \mathrm{~dB}$.

\section{CONCLUDing Remarks}

In this paper, we have derived a framework for a new class of two-channel biorthogonal filter banks. The filter banks under

Fig. 17. (Example 5.2) Magnitude responses of the perfect reconstruction FIR analysis bank: (a) $H_{0}\left(z_{0}, z_{1}\right)$; (b) $H_{1}\left(z_{0}, z_{1}\right)$.

the framework allow a structurally perfect reconstruction implementation as in Fig. 2. It is interesting that we can arrive at precisely the same ladder in Fig. 2 by using the novel approach in [26] developed for a totally different application, namely cancellation of roundoff error. The proposed systems have very low complexity. Filter banks of high frequency selectivity can be achieved by controlling a single transfer function $\beta(z)$ in Fig. 2. Two different choices of $\beta(z)$ lead to causal stable IIR and linear phase FIR filter banks, respectively. The properties of the proposed filter banks were discussed in detail. We showed that zeros at aliasing frequency can be imposed. Two new types of IIR maximally flat filters were derived and the solutions were given in closed form. In addition to perfect reconstruction property, these IIR filters have nearly linear phase in the passband. Furthermore, we also mapped the 1$D$ filter banks derived in this paper into 2-D cases. The design of a 2-D biorthogonal (stable IIR or linear phase FIR) filter bank reduces to the design of a single 1-D transfer function. 


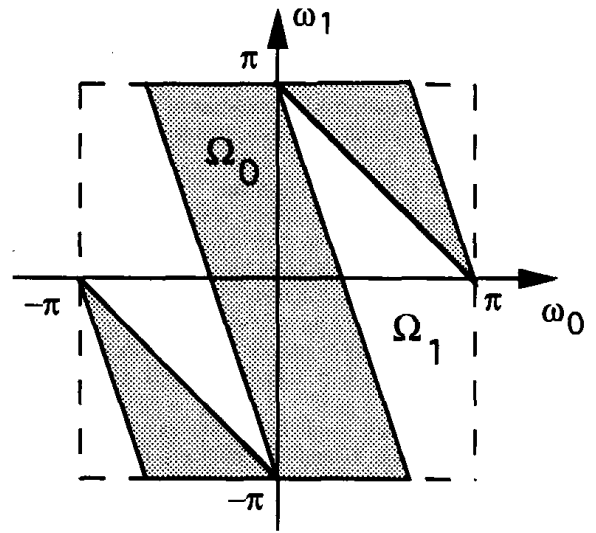

Fig. 18. Ideal supports for alias-free decimation for $\mathbf{M}$ defined in (38).

The new transformation preserves many of the properties of the 1-D systems. Before we conclude the paper, we would like to provide an example to demonstrate that the mapping in Section $\mathrm{V}$ can be easily generalized to arbitrary dilation matrix $M$ with determinant equal to 2 .

Example 6.1-2-D IIR Filter Banks: The 1-D prototype filter bank is taken to be that in Example 3.1. The dilation matrix and the coset vectors are respectively:

$$
\mathbf{M}=\left(\begin{array}{ll}
3 & 1 \\
1 & 1
\end{array}\right), \quad \mathbf{k}_{\mathbf{0}}=\left(\begin{array}{l}
0 \\
0
\end{array}\right) \quad \mathbf{k}_{\mathbf{1}}=\left(\begin{array}{l}
2 \\
1
\end{array}\right) .
$$

With the above matrix and coset vectors, the ideal passband support for the $H_{0}\left(z_{0}, z_{1}\right)$ is $\operatorname{SPD}\left(\pi \mathbf{M}^{-T}\right)$, which is shown in Fig. 18 (shaded area). By using the transformation introduced in Section $\mathrm{V}$, we find that the polyphase matrices in this example are the same as those in Example 5.1. Thus, it also has very low complexity. The only differences are the dilation matrix and the coset vectors. With the $\mathbf{M}$ and $\mathbf{k}_{\mathbf{i}}$ chosen as (38), the responses of $H_{0}\left(z_{0}, z_{1}\right)$ and $H_{1}\left(z_{0}, z_{1}\right)$ are shown in Fig. 19(a) and (b). We see that $H_{0}$ and $H_{1}$ have approximately the desired support.

\section{APPENDIX A}

PROOF OF (24)

It is shown in [33] that there exists closed form solution for $a_{N, k}$ satisfying system of linear equations:

$$
1+\sum_{k=1}^{N} a_{N, k} x_{k}^{2 r}=0, \quad \text { for } r=1,2, \cdots, N .
$$

With some modification, the solution to (23) can be written as

$$
a_{N, k}=-\frac{1}{x_{k}} \prod_{i=1}^{k-1} \frac{1-x_{k-i}^{2}}{x_{k}^{2}-x_{k-i}^{2}} \prod_{j=k+1}^{N} \frac{x_{j}^{2}-1}{x_{j}^{2}-x_{k}^{2}}
$$

where $x_{k}=1-4 k$. Substituting the value for $x_{k}$ into the equation, we find that

$$
\prod_{i=1}^{k-1} \frac{1-x_{k-i}^{2}}{x_{k}^{2}-x_{k-i}^{2}}=(-1)^{k-1} \prod_{i=1}^{k-1} \frac{(2 i-1)}{(4 k-2 i-1)},
$$

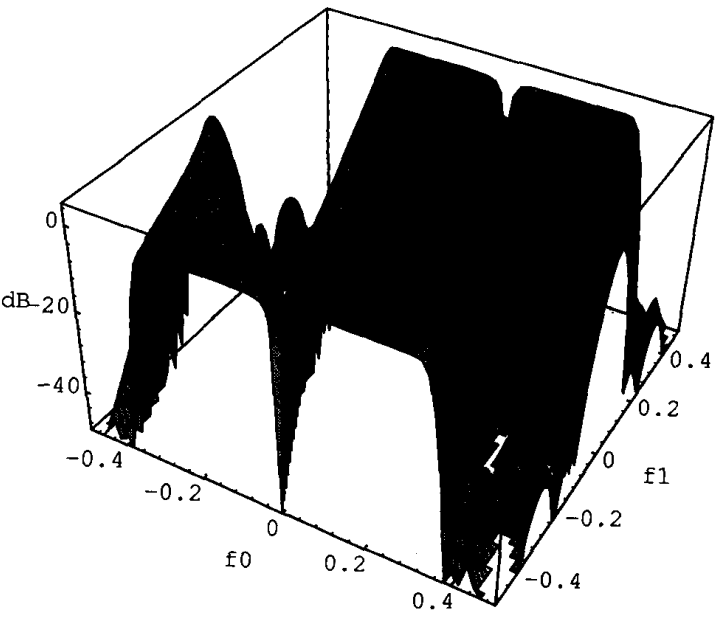

(a)

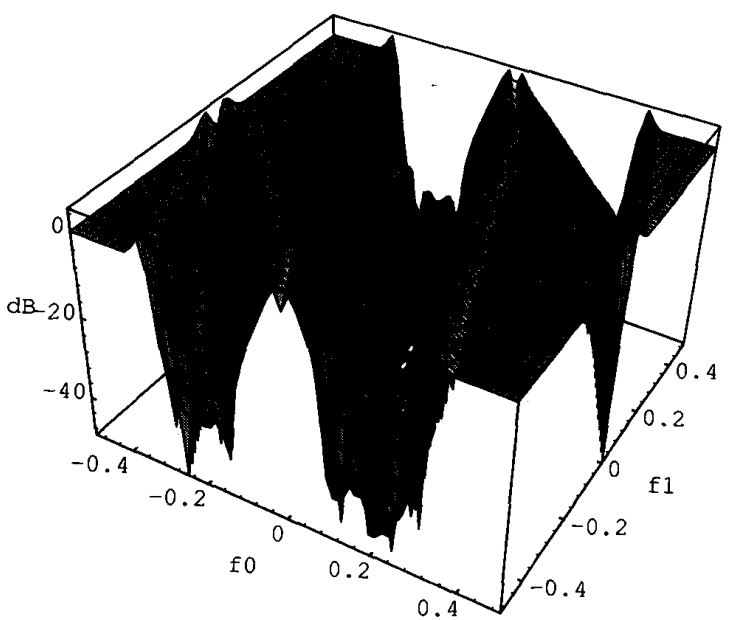

(b)

Fig. 19. (Example 6.1) Magnitude responses of the perfect reconstruction analysis bank with the decimator $\mathbf{M}$ defined in (38): (a) $H_{0}\left(\approx_{0}, \approx_{1}\right)$; (b) $H_{1}\left(\approx 0, z_{1}\right)$

$$
\prod_{j=k+1}^{N} \frac{x_{j}^{2}-1}{x_{j}^{2}-x_{k}^{2}}=\left(\begin{array}{c}
N \\
k
\end{array}\right) \prod_{j=k+1}^{N} \frac{(2 j-1)}{(2 k+2 j-1)} .
$$

Combining (A.3a) and (A.3b), we get (24).

APPENDIX B

PROOF OF PROPERTY 4 IN SECTION V-A

Supposing that the 1-D filter $H_{0}(z)$ has $k$ zeros at $\pi$, then we have

$$
e^{j \omega} H_{0}\left(e^{j \omega}\right)=e^{(-2 N+1) j \omega}+\beta\left(e^{2 j \omega}\right)=\left(1+e^{j \omega}\right)^{k} p\left(e^{j \omega}\right)
$$

where $|p(-1)|$ is a finite nonzero constant. From (32a), we have

$$
\begin{aligned}
e^{j \omega_{0}} H_{0}\left(e^{j \omega_{0}}, e^{j \omega_{1}}\right)= & e^{(-2 N+1) j \omega_{0}} \\
& +\beta\left(e^{j\left(\omega_{0}+\omega_{1}\right)}\right) \beta\left(e^{j\left(\omega_{0}-\omega_{1}\right)}\right) .
\end{aligned}
$$


From (B.2), $e^{j \omega_{0}} H_{0}\left(e^{j \omega_{0}}, e^{j \omega_{1}}\right)$ can be rewritten as

$$
\begin{aligned}
& e^{j \omega_{0}} H_{0}\left(e^{j \omega_{0}}, e^{j \omega_{1}}\right)=\beta\left(e^{j\left(\omega_{0}-\omega_{1}\right)}\right) \\
& \quad \times\left(e^{(-2 N+1) j \frac{\omega_{0}+\omega_{1}}{2}}+\beta\left(e^{j\left(\omega_{0}+\omega_{1}\right)}\right)\right)-e^{(-2 N+1) j \frac{\omega_{0}+\omega_{1}}{2}} \\
& \quad \times\left(\beta\left(e^{j\left(\omega_{0}-\omega_{1}\right)}\right)-e^{(-2 N+1) j \frac{\omega_{0}-\omega_{1}}{2}}\right)
\end{aligned}
$$

By using (B.1) and the fact that $\beta\left(e^{j \omega}\right)$ is of period $2 \pi$, we get (34).

\section{ACKNOWLEDGMENT}

The authors would like to thank Dr. T. Chen of AT\&T Bell Labs, and I. Djokovic, graduate student of California Institute of Technology, Pasadena, for fruitful discussions. Dr. Chen also provided the software for the allpass design using the eigenfilter approach for Example 3.1.

\section{REFERENCES}

[1] M. J. T. Smith and T. P. Barnwell, "A new filter-bank theory for time-frequency representation," IEEE Trans. Acoust. Speech Signal Processing, pp. 314-327, Mar. 1987.

[2] P. P. Vaidyanathan, Multirate Systems and Filter Banks. Englewood Cliffs, NJ: Prentice Hall, 1993.

[3] P. P. Vaidyanathan, "Multirate digital filters, filter banks, polyphase networks and applications: a tutorial," Proc. IEEE, vol 78, pp. 56-93, Jan. 1990.

[4] M. Vetterli, "A theory of multirate filter banks," IEEE Trans. Acoust. Speech Signal Processing, pp. 356-372, Mar. 1987.

[5] P. P. Vaidyanathan, "Orthonormal and biorthonormal filter-banks as convolvers, and convolutional coding gain," IEEE Trans. Signal Processing, vol. 41, pp. 2210-2231, June 1993.

[6] I. Daubechies, "Orthonormal bases of compactly supported wavelets," Commun. Pure Appl. Math., vol. 41, pp. 909-996, Nov. 1988.

[7] P. P. Vaidyanathan, P. Regalia, and S. K. Mitra, "Design of doubly complementary IIR filters using a single complex allpass filter, with multirate applications," IEEE Trans. Circ. Syst., vol. CAS-34, pp. 378-389, Apr. 1987.

[8] T. A. Ramstad, "IIR filter bank for subband coding of images," in Proc. IEEE Int. Symp. Circ. Syst., Espoo, Finland, 1988, pp. 827-830.

[9] C. Herley and M. Vetterli, "Wavelets and recursive filter banks," IEEE Trans. Signal Processing, vol. 41, pp. 2536-56, Aug. 1993.

[10] S. K. Mitra, C. D. Creusere, and H. Babic, "A novel implementation of perfect reconstruction QMF banks using IIR filters for infinite length signals," in Proc. IEEE Int. Symp. Circ. Syst., San Diego, CA, May 1992, pp. 2312-15.

[11] S. Basu, C.-H. Chiang, and H. M. Choi, "Wavelets and perfect reconstruction subband coding with causal stable IIR filters," preprint.

[12] E. Viscito and J. Allebach, "Design of perfect reconstruction multidimensional filter banks using cascaded Smith form matrices," Proc. IEEE Int. Symp. Circ. Syst., Espoo, Finland, June 1988, pp. 831-834.

[13] G. Karlsson and M. Vetterli, "Theory of two-dimensional multirate filter banks," IEEE Trans. Acoust. Speech Signal Processing, vol. 38, pp. 925-937, June 1990

[14] I. A. Shah and A. A. C. M. Kalker, "Generalized theory of multidimensional $M$-band filter bank design," in Proc. 6th EUSIPCO, Aug. 1992, pp. 969-972.

[15] R. Ansari and C.-L. Lau, "Two-dimensional IIR filters for exact reconstruction in tree-structured subband decomposition," Elect. Lett., vol. 23, pp. 633-634, June 1987.

[16] T. Chen and P. P. Vaidyanathan, "Multidimensional multirate filters and filter banks derived from one-dimensional filters," IEEE Trans. Signal Processing, vol. 41, pp. 1749-65, May 1993.

[17] A. Cohen and I. Daubechies, "Non-separable bidimensional wavelet bases," preprint.

[18] B. H. Tay and N. G. Kingsbury, "Flexible design of multidimensional perfect reconstruction FIR 2-band filters using transformations of variables," IEEE Trans. Image Processing, vol. 2, pp. 466-480, Oct. 1993.

[19] J. Kovacevic and M. Vetterli, "Nonseparable multidimensional perfect reconstruction filter banks and wavelet bases for $R^{\prime \prime}$, IEEE Trans. Inform. Theory, vol. 38, pp. 533-555, Mar. 1992.
[20] C. W. Kim and R. Ansari, "FIR/IIR exact reconstruction filter banks with applications to subband coding of images," in Proc. Midwest CAS Symp., Monterey, CA, May 1991.

[21] "Subband decomposition procedure for quincunx sampling grids," in Proc. SPIE-Visual Commun. Image Processing, Boston, MA, Nov. 1991.

[22] S.-M. Phoong and P. P. Vaidyanathan, "Two-channel 1-D and 2-D biorthonormal filter banks with causal stable IIR and linear phase FIR filters," in Proc. IEEE Int. Symp. Circ. Syst., London, U.K., May 1994.

[23] H. Kiya, M. Yae, and M. Iwahashi, "A linear phase two-channel filter bank allowing perfect reconstruction," in Proc. IEEE Int. Symp. Circ. Syst., San Diego, CA, 1992, pp. 951-954.

[24] 0 . Herrmann, "On the approximation problem in nonrecursive digital filter design," IEEE Trans. Circ. Theory, vol. CT-18, pp. 411-413, May 1971.

[25] R. Ansari, C. Guillemot, and J. F. Kaiser, "Wavelet construction using Lagrange halfband filters," IEEE Trans. Circ. Syst., vol. 38, pp. 1116-1118, Sept. 1991.

[26] A. M. Bruekers and Adm. W. M. van den Enden, "New networks for perfect inversion and perfect reconstruction," IEEE J. Select. Areas Commun., vol. 10, pp. 130-137, Jan. 1992.

[27] R. Ansari and B. Liu, "Efficient sampling rate alteration using recursive (IIR) digital filters," IEEE Trans. Acoust. Speech Signal Processing, vol. ASSP-31, pp. 1366-73, Dec. 1983.

[28] M. Renfors and T. Saramaki, "Recursive $N$ th-band digital filters-Part I: Design and properties," IEEE Trans. Circ. Syst., vol. 34, no. 1, pp. 24-39, Jan. 1987.

[29] T. Q. Nguyen, T. I. Laakso and R. D. Koilpillai, "Eigenfilter approach for the design of allpass filters approximating given phase response," preprint.

[30] P. P. Vaidyanathan and T. Q. Nguyen, "A "TRICK" for the design of FIR half-band filters," IEEE Trans. Circ. Syst., vol. CAS-34, pp. 297-300, Mar. 1987.

[31] R. E. Crochiere and L. R. Rabiner, Multirate Digital Signal Processing. Englewood Cliffs, NJ: Prentice Hall, 1983.

[32] T. Chen and P. P. Vaidyanathan, "Design of IFIR eigenfilters," in Proc. IEEE Int. Symp. Circ. Syst., Singapore, 1991, pp. 264-267.

[33] C. Gumacos, "Weighting coefficients for certain maximally flat nonrecursive digital filters," IEEE Trans. Circ. Syst., vol. CAS-25, pp. 234-235, Apr. 1978.

[34] I. Daubechies, Private communication.

[35] N. K. Bose, Applied Multidimensional Systems Theory. New York: Van Nostrand Reinhold, 1982.

[36] S. Lang, Calculus of Several Variables. New York: Springer-Verlag, 1987.

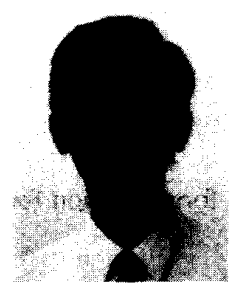

See-May Phoong (S'93) received the B.S. degree in electrical engineering from the National Taiwan University, Taipei, Taiwan, in 1991 and the M.S. degree in electrical engineering from the California Institute of Technology in 1992. He is currently pursuing the doctoral degree at the California Institute of Technology, Pasadena.

His research interests include digital signal processing, multirate filter.banks, and wavelet transforms.

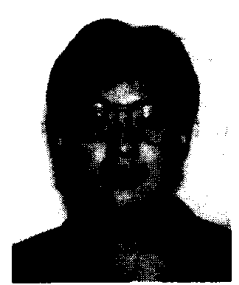

Chai W. Kim received the B.S. degree in 1981 and the M.S. degree in 1986 in electrical engineering from the University of Pennsylvania. Presently, he is working towards the Ph.D. degree in electrical engineering at the University of Pennsylvania, Philadelphia.

He worked at Burroughs Corporation from 1981 to 1984 . 


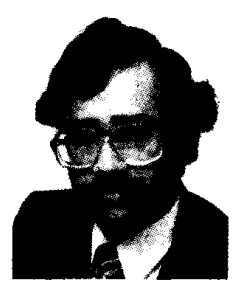

P. P. Vaidyanathan (S'80-M'83-SM'88-F'91) received the B.Sc. (Hons.) degree in physics and the B.Tech. and M.Tech. degrees in radiophysics and electronics, all from the University of Calcutta, India, in 1974, 1977, and 1979, respectively, and the $\mathrm{Ph}$. D. degree in electrical and computer engineering from the University of California at Santa Barbara in 1982 .

He was a post doctoral fellow at the University of California, Santa Barbara, from September 1982 to March 1983. In March 1983, he joined the Electrical Engineering Department of the California Institute of Technology, Pasadena, as an Assistant Professor and, since 1983, has been a Professor of Electrical Engineering there. His main research interests are in digital signal processing, multirate systems, wavelet transforms, and adaptive filtering

Dr. Vaidyanathan served as Vice-Chairman of the Technical Program Committee for the 1983 IEEE International Symposium on Circuits and Systems and as the Technical Program Chairman for the 1992 IEEE International Symposium on Circuits and Systems. He was an Associate Editor for the IEEE TRANSACTIONS ON CIRCUITS AND SYSTEMS for the period of 1985-1987, is currently an Associate Editor of IEEE Signal Processing LeTters, and is a consulting editor for the journal Applied and Computational Harmonic Analysis. He has authored a number of papers in IEEE journals and is the author of the book Multirate Systems and Filter Banks (Englewood Cliff, NJ: Prentice Hall, 1993). He has written several chapters for various signal processing handbooks. He was the recipient of the Award for Excellence in Teaching at the California Institute of Technology for 1983-1984, 1992-1993, and 1993-1994. He also received the NSF's Presidential Young Investigator Award in 1986. In 1989, he received the IEEE ASSP Senior Award for his paper on multirate perfect-reconstruction filter banks. In 1990, he was the recipient of the S. K. Mitra Memorial Award from the Institute of Electronics and Telecommunications Engineers, India, for his joint paper in the IETE journal. He was also the coauthor of a paper on linear-phase perfectreconstruction filter banks in the IEEE Transactions on Signal Processing, for which the first author (T. Nguyen) received the Young Outstanding Author Award in 1993.

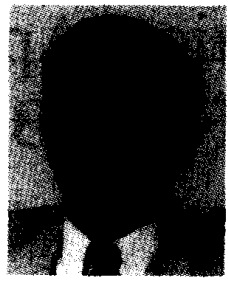

Rashid Ansari received the B.Tech. and M.Tech degrees in electrical engineering from the Indian Institute of Technology, Kampur, India, in 1975 and 1977, respectively. He received the Ph.D. degree in electrical engineering and computer science from Princeton University, NJ, in 1981.

Currently, he is a Member of the Technical Staff at Bell Communications Research, Morristown, NJ, where he has worked in the areas of image and video coding and transmission, multirate digital signal processing, and speech processing. He has served as adjunct faculty at Polytechnic University, Brooklyn, NY. Prior to that he served on the faculty of the Electrical Engineering Department at the University of Pennsylvania, Philadelphia, where he was involved in research in the areas of digital signal processing, signal reconstruction, and spectrum estimation.

Dr. Ansari was an Associate Editor of IEEE TRANSACTIONS ON CIRCUITS AND SYSTEMS during 1987-1989 and is a member of the Digital Signal Processing Technical Committee of the Circuits and Systems Society. He was a member of the editorial board of the Journal of Visual Communication and Image Representation during 1989-1992. He is currently an Associate Editor of IEEE Signal Processing LetTers. 